(c) 2006 International Press

Adv. Theor. Math. Phys. 10 (2006) 345-394

\title{
Flux compactifications on projective spaces and the $S$-duality puzzle
}

\author{
Peter Bouwknegt ${ }^{1,2}$, Jarah Evslin ${ }^{3,4}$, Branislav Jurčo ${ }^{5,6}$, \\ Varghese Mathai ${ }^{7}$ and Hisham Sati ${ }^{7,8}$ \\ ${ }^{1}$ Department of Theoretical Physics, Research School of Physical Sciences \\ and Engineering, The Australian National University, Canberra, \\ ACT 0200, Australia \\ ${ }^{2}$ Department of Mathematics, Mathematical Sciences Institute, \\ The Australian National University, Canberra, ACT 0200, Australia \\ peter.bouwknegt@anu.edu.au \\ ${ }^{3}$ International Solvay Institutes, Université Libre de Bruxelles, \\ C.P. 231, B-1050, Bruxelles, Belgium \\ ${ }^{4}$ Physique Théorique et Mathématique, Université Libre de Bruxelles, \\ C.P. 231, B-1050, Bruxelles, Belgium \\ jevslin@ulb.ac.be \\ ${ }^{5}$ Max-Planck-Institut fur Physik, Universitat Munchen, \\ Fohringer Ring 6, D-80805, Munchen, Germany \\ ${ }^{6}$ Sektion Physik, Universitat Munchen, Theresienstr. 37, D-80333, \\ Munchen, Germany \\ jurco@theorie.physik.uni-muenchen.de \\ ${ }^{7}$ Department of Pure Mathematics, University of Adelaide, \\ Adelaide, SA 5005, Australia \\ mathai.varghese@adelaide.edu.au \\ e-print archive: http://lanl.arXiv.org/abs/hep-th/0501110
}


${ }^{8}$ Department of Physics and Mathematical Physics, University of Adelaide, Adelaide, SA 5005, Australia

hisham.sati@adelaide.edu.au

\begin{abstract}
We derive a formula for D3-brane charge on a compact spacetime, which includes torsion corrections to the tadpole cancellation condition. We use this to classify D-branes and Ramond-Ramond fluxes in type II string theory on $\mathbb{R} P^{3} \times \mathbb{R} P^{2 k+1} \times S^{6-2 k}$ with torsion $H$-flux and to demonstrate the conjectured $T$-duality to $S^{3} \times S^{2 k+1} \times S^{6-2 k}$ with no flux. When $k=1, H \neq 0$ and so the $K$-theory that classifies fluxes is twisted. When $k=2$, the square of the $H$-flux yields an $S$-dual FreedWitten anomaly, which is canceled by a D3-brane insertion that ruins the dual $K$-theory flux classification. When $k=3$, the cube of $H$ is nontrivial and so the D3 insertion may itself be inconsistent and the compactification unphysical. Along the way we provide a physical interpretation for the Atiyah-Hirzebruch spectral sequence in terms of the boundaries of branes within branes.
\end{abstract}

\title{
1 Introduction
}

\subsection{What is classified by twisted $K$-theory?}

If we compactify type II string theory on a compact manifold the consistency of the D-brane partition functions implies that the Ramond-Ramond (RR) field strengths, which we write locally as $G_{p}=d C_{p-1}$, are quantized. This may lead us to believe that if we are not interested in changes by globally defined connections $C_{p-1}$ then the $G_{p}$ are classified by integral cohomology.

However several authors [1-3] have suggested that instead RR field strengths are classified by twisted $K$-theory, which is a quotient of a subset of integral cohomology. For example, all of the field strengths that are in this subset satisfy

$$
d_{3} G_{p}=\left(S q^{3}+H \cup\right) G_{p}=0,
$$

where $S q^{3}$ is an operator that takes torsion $p$-classes to torsion $(p+3)$ classes $^{1}$. In addition twisted $K$-theory identifies cohomology classes that differ by an element in the image of $d_{3}$, which is just another consequence of forgetting the globally defined connections [5].

To see what is so special about the subset (1.1), we turn our attention to the classical limit, type II supergravity. This means that we forget the

\footnotetext{
${ }^{1}$ We do not assume that the reader is familiar with the Steenrod squares $S q^{i}$. However a crash course may be found in [4].
} 
quantization condition, so we are now looking at real cohomology and the torsion is gone. In particular we can no longer see the $S q^{3}$ term. This theory has RR potentials $C_{p-1}$, an Neveu-Schwarz (NS) 3 -form $H$ and a peculiar gauge-invariance

$$
C_{p-1} \longrightarrow C_{p-1}+d \Lambda_{p-2}+H \wedge \Lambda_{p-4}
$$

for any set of forms $\Lambda_{k}$. This means that there are two natural field strengths [6]

$$
G_{p}=d C_{p-1} \quad \text { and } \quad F_{p}=d C_{p-1}+H \wedge C_{p-3},
$$

of which $G_{p}$ is closed and $F_{p}$ is gauge-invariant. As in QED, we introduce charges as violations of the Bianchi identity

$$
Q_{\mathrm{D}(8-p)}=d d C_{p-1}=d F_{p}-d\left(H \wedge C_{p-3}\right)=d F_{p}+H \wedge F_{p-2},
$$

where in the last step we have used the fact that $H \wedge H$ vanishes classically, although in the quantum theory it may have a torsion contribution. Note that this notion of D-brane charge is not the notion of D-brane charge of, for example, Ref. [7]. There the authors define D-brane charge to instead be $d F$, and refer to $d G$ as the charge contribution from local sources.

The classical limit of the condition (1.1) is $H \wedge G=0$ but instead classical supergravity yields

$$
H \wedge G_{p-2}=H \wedge F_{p-2}=Q_{\mathrm{D}(8-p)}-d F_{p} .
$$

Thus twisted $K$-theory seems to classify only fluxes in the subset of configurations for which the right hand side of equation (1.5) vanishes. There are many consistent string backgrounds that do not satisfy the condition (1.1), these correspond to cohomology classes but not to $K$-theory classes. That is, $K$-theory classifies only those configurations in which all of the branes are sources for the gauge-invariant field strength $F_{p}$ and not branes created from $H \wedge F$, such as those constructed during Hanany-Witten transitions [8]. This is not to say that the two types of branes have physically different properties, but rather that the $K$-theory formalism treats them differently. In particular, if our spacetime $M$ is compact and has no boundary, as it will be during most of this paper, then because $F_{p}$ is gauge-invariant Stokes theorem tells us that

$$
\int_{M} d F_{p}=\int_{\partial M} F_{p}=0
$$

Therefore in the compact case the right hand side of equation (1.5) vanishes only when the D-brane charge vanishes, at least up to the torsion terms that we have been neglecting. This leads us to the claim

Claim. On compact spacetimes fluxes can be classified by twisted K-theory only if the total D-brane charge is torsion. 
We will see examples in which compact spacetimes may have torsion $D$-brane charge which is non-vanishing after the torsion corrections that we will describe momentarily. Twisted $K$-theory will not classify fluxes in these cases.

While twisted $K$-cohomology classifies fluxes, twisted $K$-homology classifies the branes that source these fluxes. That is to say, it classifies the $d F$-type branes. The $H \wedge F_{p}$ type branes are quotiented away when passing from homology to $K$-homology because these branes are created by changing $F_{p}$ and the $K$-classification of branes applies only when we forget about globally defined field strengths $F_{p}$, just as the $K$-classification of fluxes required that we forget about globally defined connections $C_{p-1}$.

The analogous condition to (1.1) in the case of branes is obtained by replacing the flux $G_{p}$ with the charge $d G_{p}$. It is just the condition that the Freed-Witten anomaly [9] vanishes, or equivalently the condition that the brane is not a baryon. We will use the word baryon [10] to mean a brane on which other branes end, where the terminology came from the fact that they correspond to baryonic vortices in the worldvolume gauge theories of some probe branes.

Physical D-branes must satisfy yet another condition, which is not in general satisfied by twisted $K$-theory classes, that the flux $F$ that they source is globally defined. In particular this means that there is no net $d F$-type D-brane charge on a compact spacetime, and so the branes are not classified by twisted $K$-theory. However the twisted $K$-theory of a compact spacetime is still instructive for two reasons. First, while there is no net charge there may still be D-branes. We will see that some properties of D-branes, such as the lower brane charges that they carry and the possible remnants when they annihilate, are described by (the extension problem of) twisted $K$-theory even when the spacetime is compact. Secondly, often there is a similar configuration that is non-compact, for example in the cases $k=1$ and $k=2$ of the present paper there is an extra sphere that plays no role and may be replaced by a non-compact manifold, in which case the twisted $K$-homology classes described may yield honest D-branes.

\subsection{Powers of $H$ and the $S$-duality puzzle}

The RR gauge transformations (1.2) that lead to the twisted $K$-theory classification of RR fluxes and D-branes are not the only gauge transformations available in type II supergravities. For example, the type IIB action is invariant under $S L(2, \mathbb{R}) S$-duality transformations that mixes the RR 3 form $G_{3}$ and the NS 3-form $H$ and the $S$-duals of the gauge transformations 
(1.2) yield distinct gauge transformations. It has been conjectured [11-13] that an $S L(2, \mathbb{Z})$ subgroup is a symmetry of the full quantum theory. If this is true then one may wish to define a twisted $K$-theory corresponding to the image of equation (1.2) under each element of $S L(2, \mathbb{Z})$. Such dual $K$-classifications have been applied to stacks of D3-branes on an orientifold 3-plane in Ref. [14] and to the Klebanov-Strassler geometry [15] in Ref. [16]. While the $\left\{T^{n}\right\}=\mathbb{Z} \subset S L(2, \mathbb{Z})$ subgroup

$$
G_{3} \mapsto G_{3}+n H, \quad H \mapsto H,
$$

acts trivially on the gauge transformations and the $K$-theory classes, the orbit of the $S L(2, \mathbb{Z})$ actions on the gauge transformations still yields an infinite number of different twisted $K$-theories all of which may simultaneously classify the flux and brane spectra of a given spacetime. That is to say, a given configuration of fluxes of branes may, if it satisfies the criteria given in the last subsection, correspond to an element of each member of an infinite family of twisted $K$-theories [5]. We will refer to this family as $S$-covariant $K$-theory.

Such a configuration must satisfy an infinite number of conditions that are $S$-dual to (1.1). For example

$$
\begin{gathered}
\left(S q^{3}+G_{3}\right) H=0, \quad\left(S q^{3}+G_{3}\right) G_{5}=0, \\
\left(S q^{3}+G_{3}+H\right) G_{3}=H \cup G_{3}=0 .
\end{gathered}
$$

Note that the $\mathbb{Z} \subset S L(2, \mathbb{Z})$ subgroup that acts trivially on the gauge transformation also acts trivially on these constraints. Combining the first and last conditions of equation (1.8) with that of equation (1.1) we find in particular that

$$
G_{3} \cup G_{3}=G_{3} \cup H=H \cup H=0
$$

for all fluxes in $S$-covariant $K$-theory.

Equation (1.9) is stronger than the condition that D3-brane charge vanishes. To find this condition, we begin with a compact manifold with $H=G_{3}=0$. For simplicity we assume that the fundamental group is pure torsion so that the first cohomology group $H^{1}$ is trivial and so the field $G_{1}$ is trivial and the dilaton is globally defined. The manifold is compact and so there are no $d G_{5}$ type D3-branes, and the vanishing $G_{3}$ and $H$ insure that there are no Hanany-Witten type D3-branes. We cannot exclude the possibility that there is brane charge that results entirely from gravitational effects, which would be determined by the topology of the spacetime. For example Green-Schwarz-like terms may correct the equations of motion (1.5), such as the one-loop term in the IIA supergravity action calculated in Ref. [17] which contributes to the fundamental string charge [18]. Note that simply dualizing that contribution to obtain gravitational D-brane charge fails as fluxes are inevitably produced by the duality, but an implicit formula 
obeyed by the gravitational charge appears in Ref. [19]. We let $P$ denote the 6 -form dual to the gravitational D3-brane charge.

Now we may turn on any $H$ and $G_{3}$-flux by letting 5 -branes sweep out the dual cycles. First we turn on the $H$-flux with an NS5-brane. The NS5-brane in IIB carries a $U(1)$ gauge field under which fermions are charged that are in the spin lift of the normal bundle. If $H \cup H$ is non-vanishing then this spin lift does not exist and furthermore no shift of the $U(1)$ gauge field can render the partition function well-defined. This is the Freed-Witten anomaly [9] and it can be canceled by including a D3-brane dual to $H \cup H$ which ends on the NS5. Thus the NS5 sweeps out a cycle and vanishes, leaving behind not only the desired $H$-flux, but also $H \cup H$ units of D3 charge. Now we may turn on any $G_{3}$-flux by sweeping a D5-brane through a dual cycle. The D5-brane has a Freed-Witten anomaly $G_{3} \cup G_{3}$ resulting from its possible failure to be $\operatorname{spin}^{c}$ and a further anomaly $G_{3} \cup H$ resulting from the $H$ flux on its compact worldvolume. Again these anomalies are canceled by D3brane insertions. Thus we are able to create an arbitrary $G_{3}$ and $H$-flux, but in the process we automatically change the D3-brane charge (figure 1).

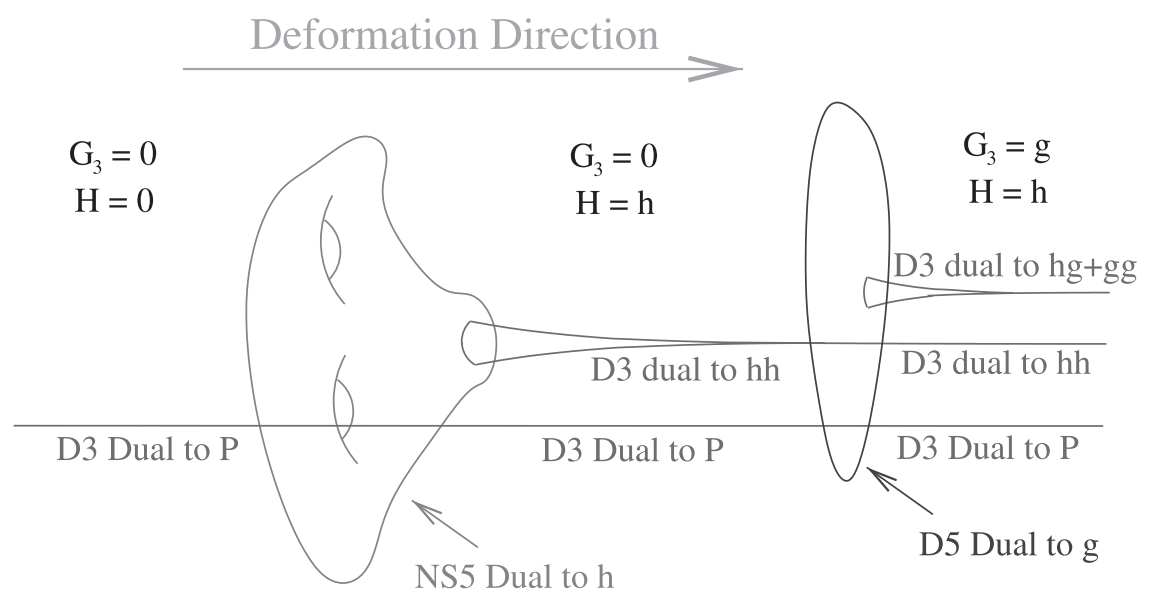

Figure 1: On the left the fluxes are turned off and the D3-brane charge $P$ is determined by the topology of the spacetime. As we move to the right, an NS5 sweeps out the cycle dual to the 3 -class $h$, and so to the right of the NS5 there is an NS flux $H=h$. The Freed-Witten anomaly of the NS5-brane is canceled by inserting a D3 dual to $h h$, and so the D3 charge changes by $h h$. Next we pass a D5-brane dual to $g$, whose Freed-Witten anomaly is $h g+g g$. The anomaly requires that the D3 charge jump by $h g+g g$. At every stage the D3 charge is $P+H H+G_{3} H+G_{3} G_{3}$, and on the right $G_{3}$ and $H$ are arbitrary. 
Claim. Type IIB on a compact spacetime with torsion fundamental group has D3 charge equal to $H \cup H+H \cup G_{3}+G_{3} \cup G_{3}+P$.

This claim extends a result proven in Ref. [19] to more general backgrounds, in particular to backgrounds with D-branes and to backgrounds not dual to $M$-theory compactified on a 2-torus. Note that while $H \cup G_{3}$ is the familiar term from tadpole cancellation in the flux compactifications literature, this claim suggests that the other three terms appear as torsion corrections to the usual formula. This would kill most flux vacua. However, it is possible that it would not change scales by enough orders of magnitude to be detected by landscape considerations.

If the D3-brane itself wraps nontrivial $G_{3}$ or $H$-flux then it also suffers from a Freed-Witten anomaly, which must be canceled by inserting F-strings or D-strings respectively. The string worldsheets are tubes with one end wrapping the dual of the offending flux in the D3 worldvolume. The spacetime is compact and so the string must have two ends. The other end of the string must be on a second brane, where it will act as some kind of source in the worldvolume gauge theory. This second brane is compact, as the spacetime is compact, and so the charge needs to be canceled by a worldvolume flux on the second brane. The compactness of spacetime also means that the second brane cannot be a source brane, but rather is a Hanany-Witten type brane.

If for example the string is an F-string then the other end may be on a second brane, which is a D1 that wraps a cycle with $G_{1}$ flux or else a D5 that wraps a cycle with a five-form flux. The second brane is not a source brane, and so in particular the D5 must satisfy

$$
Q_{\mathrm{D} 5}=H \cup G_{1} \text {. }
$$

In either case we see that $G_{1}$ needs to be nontrivial for this cancellation to occur, and so the spacetime must have $H^{1} \neq 0$ and so the fundamental group must not be pure torsion. Thus if the fundamental group is torsion then there is no candidate for the second brane and so the anomaly cannot be canceled. Therefore the D3-charge cupped with $G_{3}$ and $H$ must vanish, which leads to our final claim

Claim. Type IIB on a compact spacetime with torsion fundamental group is consistent only if $G_{3} \cup G_{3} \cup G_{3}+G_{3} \cup P$ and $H \cup H \cup H+H \cup P$ vanish.

In this paper we will test these claims by considering three examples of type IIB string theory backgrounds, $\mathbb{R} P^{3} \times \mathbb{R} P^{2 k+1} \times S^{6-2 k}$ with torsion $H$ flux, which have $H \neq 0, H \cup H \neq 0$ and $H \cup H \cup H \neq 0$ for $k=1,2$ 
and 3, respectively. These backgrounds are particularly simple to understand because [20], as we will show in Section 4, they are $T$-dual to $S^{3} \times$ $S^{2 k+1} \times S^{6-2 k}$ with no fluxes at all. The example $k=2$ is not spin, and so the definition of string theory on this space is not obvious, although one might guess that the $H$ flux provides some kind of generalized $\operatorname{spin}^{c}$ structure. This is the case after dimensionally reducing on a two-torus, the two 2 -forms resulting from the dimensional reduction of $H$ are the curvatures of the two dual circle bundles whose total space is spin. As in Ref. [21], the $T$-duality to $S^{3} \times S^{5} \times S^{2}$ with no flux may imply that the string theory is well defined and even supersymmetric via "supersymmetry without supersymmetry". However [20], as in [21], the dual spheres are smaller than the string scale and so it is not clear that they provide a definition. Instead it may be necessary to define this background as an orientifold. In this case some fields will be valued in $\mathbb{Z}_{2}$ twisted cohomology [10,22].

We will describe the D3-brane insertions in Section 5 and then in Section 6 we will use the $T$-duality prescription of Refs. $[23,24]$ to follow these and other D-branes through the $T$-dualities. This allows us to compare the above conjectures about Freed-Witten anomalies on projective spaces to the better understood physics of the compactification on a product of spheres, where the absence of torsion and of fluxes means that none of these anomalies are present.

A central role is played by the twisted $K$-theory classification of branes. In Section 3 we will use the Atiyah-Hirzebruch spectral sequence (AHSS) to compute the twisted $K$-theories of each space and demonstrate that as expected $T$-duality shifts the dimension by one. This map on twisted $K$-theory will then allow us to confirm that we have correctly $T$-dualized each of the branes. Many of these computations are new and we hope that they will be of independent interest.

Along the way we will run into a number of interesting phenomena. For example in Section 2 we will, following the suggestion of Ref. [25], identify the lower brane charges carried by D-branes with the solution to the $K$ theory extension problem. In particular we will see an example in which a lower half-brane charge is carried when the normal bundle is spin and the $B$-field is trivial, which is possible because of a factor of two that appears in the spectral sequence. In Section 4 we will find an example in which the differential $d_{5}$ acts nontrivially, and we will see that its action agrees with the conjectured form of $d_{5}$ in Ref. [26]. We will also find, in Section 6, a composite Freed-Witten anomaly that creates a topologically nontrivial charge from a topologically trivial flux (although the deformation to the trivial flux does not respect a free circle action) and a gauge-dependent 


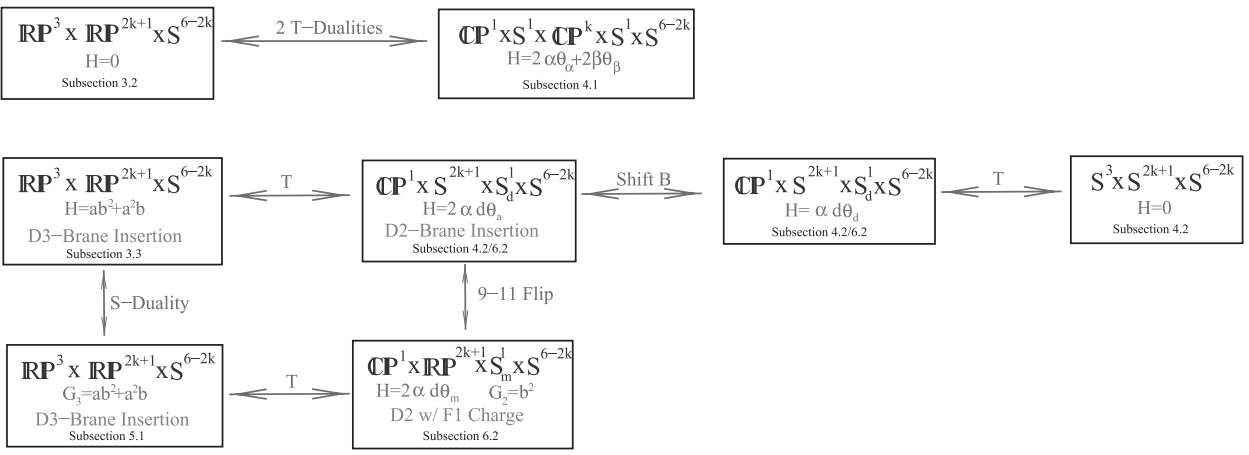

Figure 2: This is a summary of the main compactifications that appear in this note and the dualities and transformations that relate them.

flux. We will use this anomaly to partially extend the Diaconescu-MooreWitten (DMW) [4] anomaly $W_{7}=0$ to the non-spin case. In the appendix we summarize the relevant properties of projective spaces (figure 2).

\section{Warm up: type IIB on $\mathbb{R} P^{7} \times S^{3}$ without flux}

In this section we will classify RR fluxes and branes in type IIB string theory on $\mathbb{R} P^{7} \times S^{3}$ with no NS flux. The relation between branes of various dimensions that will be crucial in the main argument of this note may already be seen in this simpler example. We begin by using the AHSS and Künneth theorem to review the relevant untwisted $K$-theories. Then we interpret these groups physically in terms of fluxes and also in terms of branes carrying the charges of lower-dimensional branes.

\subsection{Calculating untwisted $K$-groups}

In Ref. [27] Atiyah and Hirzebruch showed that the associated graded $K$ theory of a space $X, \operatorname{Gr}(K(X))$, which is related to $K$-theory by an extension problem, may be calculated from the cohomology of $X$ by taking a series of subsets and quotients. In this example, unlike the examples that we will encounter later, this process is trivial and $\operatorname{Gr}(K(X))$ is in fact just the original integral cohomology, or more precisely the sum of either the even 
or the odd cohomology. The relevant homology and cohomology groups are

$$
\begin{gathered}
H_{0}\left(S^{3}\right)=H_{3}\left(S^{3}\right)=H_{0}\left(\mathbb{R} P^{7}\right)=H_{7}\left(\mathbb{R} P^{7}\right)=\mathbb{Z}, \\
H_{1}\left(\mathbb{R} P^{7}\right)=H_{3}\left(\mathbb{R} P^{7}\right)=H_{5}\left(\mathbb{R} P^{7}\right)=\mathbb{Z}_{2}, \\
H^{0}\left(S^{3}\right)=H^{3}\left(S^{3}\right)=H^{0}\left(\mathbb{R} P^{7}\right)=H^{7}\left(\mathbb{R} P^{7}\right)=\mathbb{Z}, \\
H^{2}\left(\mathbb{R} P^{7}\right)=H^{4}\left(\mathbb{R} P^{7}\right)=H^{6}\left(\mathbb{R} P^{7}\right)=\mathbb{Z}_{2},
\end{gathered}
$$

and so the associated graded $K$-groups are

$$
\begin{gathered}
\operatorname{Gr}\left(K_{0}\left(S^{3}\right)\right)=\operatorname{Gr}\left(K_{1}\left(S^{3}\right)\right)=\operatorname{Gr}\left(K^{0}\left(S^{3}\right)\right)=\operatorname{Gr}\left(K^{1}\left(S^{3}\right)\right)=\mathbb{Z}, \\
\operatorname{Gr}\left(K_{0}\left(\mathbb{R} P^{7}\right)\right)=\mathbb{Z}, \quad \operatorname{Gr}\left(K_{1}\left(\mathbb{R} P^{7}\right)\right)=\mathbb{Z} \oplus \mathbb{Z}_{2}^{3}, \\
\operatorname{Gr}\left(K^{0}\left(\mathbb{R} P^{7}\right)\right)=\mathbb{Z} \oplus \mathbb{Z}_{2}^{3} \quad \operatorname{Gr}\left(K^{1}\left(\mathbb{R} P^{7}\right)\right)=\mathbb{Z} .
\end{gathered}
$$

To obtain the actual $K$-groups we need to solve a series of extension problems. The ones that will be nontrivial are described by the short exact sequences

$$
\begin{aligned}
& H_{1}\left(\mathbb{R} P^{7}\right)=\mathbb{Z}_{2} \rightarrow F_{2} \rightarrow H_{3}\left(\mathbb{R} P^{7}\right)=\mathbb{Z}_{2}, \quad F_{2} \rightarrow F_{3} \rightarrow H_{5}\left(\mathbb{R} P^{7}\right)=\mathbb{Z}_{2}, \\
& H^{6}\left(\mathbb{R} P^{7}\right)=\mathbb{Z}_{2} \rightarrow F^{2} \rightarrow H^{4}\left(\mathbb{R} P^{7}\right)=\mathbb{Z}_{2}, \quad F^{2} \rightarrow F^{1} \rightarrow H^{2}\left(\mathbb{R} P^{7}\right)=\mathbb{Z}_{2} .
\end{aligned}
$$

The solutions to these extension problems are not unique, and so we will state the answers which are derived in, for example, Ref. [28] and then explain the underlying physics. The solution is

$$
F_{2}=F^{2}=\mathbb{Z}_{4}, \quad F_{3}=F^{1}=\mathbb{Z}_{8},
$$

and so the three $\mathbb{Z}_{2}$ torsion groups in each $\operatorname{Gr}(K)$ assemble into a single $\mathbb{Z}_{8}$ in the actual $K$-groups

$$
\begin{gathered}
K_{0}\left(S^{3}\right)=K_{1}\left(S^{3}\right)=K^{0}\left(S^{3}\right)=K^{1}\left(S^{3}\right)=\mathbb{Z}, \\
K_{0}\left(\mathbb{R} P^{7}\right)=\mathbb{Z}, \quad K_{1}\left(\mathbb{R} P^{7}\right)=\mathbb{Z} \oplus \mathbb{Z}_{8}, \\
K^{0}\left(\mathbb{R} P^{7}\right)=\mathbb{Z} \oplus \mathbb{Z}_{8}, \quad K^{1}\left(\mathbb{R} P^{7}\right)=\mathbb{Z} .
\end{gathered}
$$

This means that if we interpret the generator $x$ of $H^{2}\left(\mathbb{R} P^{7}\right)=\mathbb{Z}_{2}$ as the generator of the $\mathbb{Z}_{8} \subset K^{0}\left(\mathbb{R} P^{7}\right)$ then in $K$-theory instead of being order $2, x$ is order 8 . Thus while $2 x \in H^{2}\left(\mathbb{R} P^{7}\right)=\mathbb{Z}_{2}$ is the trivial element in cohomology, as an element of $K$-theory it is nontrivial and corresponds to the generator $y$ of $\mathrm{H}^{4}\left(\mathbb{R} P^{7}\right)=\mathbb{Z}_{2}$, while $3 x$ in $K$-theory corresponds to $x+y$ in cohomology.

So far this is just what one would find by associating the elements of $H^{2 k}$ with the Chern classes $c_{k}$ of the corresponding $K$-class and defining addition to be the direct sum of the corresponding bundles. However this naive 
association fails for $4 x$, which corresponds to the generator $z$ of $\mathrm{H}^{6}\left(\mathbb{R} P^{7}\right)=$ $\mathbb{Z}_{2}$ in the spectral sequence. This cannot be the third Chern class because ${ }^{2}$

$$
c_{3}=c_{1} \cup c_{2}+\mathrm{sq}^{2} c_{2} \bmod 2,
$$

and so when $c_{1}=c_{2}=0$ the third class $c_{3}$ must be even and thus trivial in $H^{6}\left(\mathbb{R} P^{7}\right)=\mathbb{Z}_{2}$. We will argue that the class $z$ corresponds not to $c_{3}$ but to $c h_{3}=c_{3} / 2$ which is odd. Ordinarily, the Chern characters are only elements of rational cohomology and thus do not see torsion classes, which would mean that $c h_{3}$ ordinarily would miss the $\mathbb{Z}_{2}$. However, we claim that in the cases in which Chern characters compute charges in string theory they do admit a lift to integral cohomology (with normalization defined by the AHSS) and so are sensitive to torsion. Here this is a result of the evenness of $c_{3}$, but in general it will reflect that fact that they may be expressed as the images of products of Steenrod squares and Steenrod powers.

Similarly, the $\mathbb{Z}_{8}$ subgroup of $K_{1}\left(\mathbb{R} P^{7}\right)$ is generated by the generator $x$ of $H_{5}\left(\mathbb{R} P^{7}\right)=\mathbb{Z}_{2}$, which is order 2 in homology and order 8 in $K$-homology. $2 x$ generates $H_{3}\left(\mathbb{R} P^{7}\right)=\mathbb{Z}_{2}$ while $4 x$ generates $H_{1}\left(\mathbb{R} P^{7}\right)=\mathbb{Z}_{2}$, where again we will see a crucial factor of two.

We may now combine the $K$-theories of the sphere and projective space to find the $K$-theory of the product using the Künneth formula. The result is just the tensor product of the original $K$-theories because the $K$-theory of the sphere has no torsion and so the Tor term in the Künneth formula is trivial

$$
\begin{aligned}
& K_{0}\left(\mathbb{R} P^{7} \times S^{3}\right)=\left(K_{0}\left(\mathbb{R} P^{7}\right) \otimes K_{0}\left(S^{3}\right)\right) \oplus\left(K_{1}\left(\mathbb{R} P^{7}\right) \otimes K_{1}\left(S^{3}\right)\right)=\mathbb{Z}^{2} \oplus \mathbb{Z}_{8}, \\
& K_{1}\left(\mathbb{R} P^{7} \times S^{3}\right)=\left(K_{0}\left(\mathbb{R} P^{7}\right) \otimes K_{1}\left(S^{3}\right)\right) \oplus\left(K_{1}\left(\mathbb{R} P^{7}\right) \otimes K_{0}\left(S^{3}\right)\right)=\mathbb{Z}^{2} \oplus \mathbb{Z}_{8}, \\
& K^{0}\left(\mathbb{R} P^{7} \times S^{3}\right)=\left(K^{0}\left(\mathbb{R} P^{7}\right) \otimes K^{0}\left(S^{3}\right)\right) \oplus\left(K^{1}\left(\mathbb{R} P^{7}\right) \otimes K^{1}\left(S^{3}\right)\right)=\mathbb{Z}^{2} \oplus \mathbb{Z}_{8}, \\
& K^{1}\left(\mathbb{R} P^{7} \times S^{3}\right)=\left(K^{0}\left(\mathbb{R} P^{7}\right) \otimes K^{1}\left(S^{3}\right)\right) \oplus\left(K^{1}\left(\mathbb{R} P^{7}\right) \otimes K^{0}\left(S^{3}\right)\right)=\mathbb{Z}^{2} \oplus \mathbb{Z}_{8} .
\end{aligned}
$$

The extension problem now means that, for example, the generator

$$
x \in H^{5}\left(\mathbb{R} P^{7} \times S^{3}\right)=H^{2}\left(\mathbb{R} P^{7}\right) \otimes H^{3}\left(S^{3}\right)=\mathbb{Z}_{2},
$$

is order two in cohomology but order eight in $K$-theory. So for example $2 x$ is the generator of $H^{4}\left(\mathbb{R} P^{7}\right) \otimes H^{3}\left(S^{3}\right)$.

The untwisted $K$-theories of other $\mathbb{R} P^{2 k+1}$ 's are obtained identically, except that there are $k$ factors of $\mathbb{Z}_{2}$ and so $\mathbb{Z}_{8}$ is replaced by $\mathbb{Z}_{2^{k}}$.

\footnotetext{
${ }^{2}$ We will denote by sq ${ }^{k}$ the Steenrod square in cohomology with $\mathbb{Z}_{2}$ coefficients, and by $S q^{2 k+1}$ the lift of $\mathrm{sq}^{2 k+1}$ to integer coefficients.
} 


\subsection{Branes and fluxes on $\mathbb{R} P^{7} \times S^{3}$}

In type IIA string theory D-branes are classified by $K_{1}$ while $\mathrm{RR}$ field strengths are classified by $K^{0}$, both of which in our case are $\mathbb{Z}^{2} \oplus \mathbb{Z}_{8}$. Thus all branes and fluxes are generated by three elementary ones, the generators of the respective $K$-groups, one of which is order eight. For example the fluxes are generated by $G_{0}$, which is the Romans' mass [29], $G_{10}$, which again is the Romans' mass (in the dual sense), and finally $G_{2} \in H^{2}\left(\mathbb{R} P^{7}\right) \otimes H^{0}\left(S^{3}\right)$ which is torsion. The Romans' mass may appear twice because $K$-theory does not really classify fluxes in string theory, which cannot all be simultaneously quantized, but rather on a toy model of string theory in which the self-duality condition

$$
G_{p}=* G_{10-p}
$$

is not imposed. This omission is crucial as the Hodge star varies continuously with the metric and so is incompatible with the simultaneous quantization of the Chern characters that is inherent in the $K$-theory classification. In the physical string theory [30] only half of the fluxes may be quantized at a time, but on a compact space one needs to check that the partition function is invariant under the choice of which half is quantized, as in the case in the chiral scalar 2-dimensional field theory. Thus one should expect $K$-theory to double-count the simultaneously observable degrees of freedom.

$G_{2}$ is the Chern class of the $M$-theory circle which is fibered over $\mathbb{R} P^{7} \times$ $S^{3}$. While there are only two possible fibrations of the $M$-theory circle, corresponding to the two elements of $H^{2}$, as an element of $K$-theory if we add together the two nontrivial $M$-theory bundles we find that while the $M$-theory bundle becomes trivial, we now have a unit of $G_{4}$-flux. Similarly adding together two units of $G_{4}$-flux we find a unit of $G_{6}$. For a global version of the reduction of $M$-theory to type IIA leading to twisted $K$-theory, see [31].

Although adding fluxes may seem a bit abstract if not ill-defined, we may find the same result by physically adding two D-branes that source the corresponding fluxes. The D-branes are classified by $K_{1}=\mathbb{Z}^{2} \oplus \mathbb{Z}_{8}$ and so again there are three generators. There is a D2-brane that wraps the $S^{3}$, a D6-brane that wraps the $\mathbb{R} P^{7}$, and finally there is a torsion D4-brane that wraps the submanifold $\mathbb{R} P^{5} \subset \mathbb{R} P^{7}$. While a homology classification of D-branes would tell us that by deforming the configuration two D4-branes can annihilate to nothing, as they are each $\mathbb{Z}_{2}$ charged, in fact after the annihilation a D2-brane remains that wraps $\mathbb{R} P^{3} \subset \mathbb{R} P^{7}$. Similarly two D2-branes may annihilate to leave a D0-brane wrapping the circle $\mathbb{R} P^{1}$. However if two D0-branes annihilate, nothing remains. Thus we say that 
the D4-branes each carry half of a unit of D2-brane charge while the D2branes each carry half a unit of D0-brane charge. Note that while the element 1 corresponds to a D4-brane with half a unit of D2 charge, the unit 7 corresponds to a D4-brane with negative half a unit of D2-charge, and so the two D4-branes corresponding to 1 and 7 may annihilate to a state that contains no D2-branes. However there is no such confusion if we choose orientation conventions (the SUSYs that will be preserved by branes) and write all states in terms of branes and not antibranes, so that 7 is written as a D4-brane plus a D2-brane plus a D0.

This explains the meaning of two units of $G_{2}$-flux combining to form a unit of $G_{4}$-flux. We may consider a D6-brane wrapping $\mathbb{R} P^{5} \times S^{3}$ that sources the $G_{2}$ flux. There is no space in $\mathbb{R} P^{7} \times S^{3}$ for such a D6-brane and in fact it has one too many dimensions, however we are free to add another dimension $v$ that is parameterized by deformations $[2,32]$. In fact, one such choice of dimension that appears naturally in applications is the Renormalization Group (RG) flow [16]. That is to say that a $G_{2}$-flux measured at one energy scale (a dimensional reduction of $M$-theory defined by some characteristic distance) may differ from the $G_{2}$-flux measured at another scale, and this difference corresponds to a dynamical process ${ }^{3}$ in which a D6-brane has nucleated, swept out a linking 8-cycle, and collapsed out of existence. In the Klebanov-Strassler cascade [15], for example, the relation between the brane position and energy scale is a consequence of the RG flow of the coupling constant of the worldvolume effective gauge theory.

Thus the 10-dimensional cross-section at one value of $v$ enjoys a nontrivial $G_{2}$-flux generating $H^{2}\left(\mathbb{R} P^{7}\right)$, but as one moves in the $v$ direction one passes a D6-brane (corresponding to $1 \in \mathbb{Z}_{8}$ ), wrapping $\mathbb{R} P^{5} \times S^{3}$, and the $G_{2}$-flux turns off. One may pass a second D6-brane and the flux turns back on. If this second D6-brane corresponds to the element $1 \subset \mathbb{Z}_{8}$, and not the element $7 \subset \mathbb{Z}_{8}$ then the sum of these two D6-branes carries a unit of D4brane charge. This means that as we pass both of these branes $G_{4}$ jumps by a unit. This is the meaning of the fact that two units of $G_{2}$-flux add to a unit of $G_{4}$-flux, and also the source of the caveat that we need to be sure that our nontrivial $G_{2}$ 's both lift to the same element in $K$-theory.

\subsection{Solving the extension problem with physics}

We have argued that the physical interpretation of the nontrivial solution of the extension problem is that the D4-brane wrapping $\mathbb{R} P^{5} \subset \mathbb{R} P^{7}$ carries

\footnotetext{
${ }^{3}$ Here "time" refers to the RG direction, so for example the process may occur as the system relaxes. More generally $K$-classes appear to correspond to universality classes in the worldvolume theories.
} 
half a unit of D2 charge, while the D2 wrapping $\mathbb{R} P^{3}$ carries half a unit of D0 charge. It should be possible to understand these charges, and thus the solution to the extension problem, in terms of the worldvolume physics of the corresponding branes. For example, finding the D2 charge in the D4brane is routine. The D4-brane worldvolume theory contains fermions that are charged under the worldvolume $U(1)$ gauge field and also are valued in the spinor representation of the normal bundle. The $S^{3}$ part of the normal bundle is trivial, however the normal bundle $N$ of $\mathbb{R} P^{5} \subset \mathbb{R} P^{7}$ is not spin. This means that there is an obstruction to lifting the normal bundle to a spin bundle, and so the consistency of the fermion partition function demands that the $U(1)$ bundle enjoy an obstruction that cancels that of the normal bundle. That is to say, the quantization of the $U(1)$ gauge field is shifted

$$
F \in \mathbb{Z}+\frac{1}{2}
$$

We may now use the D4-brane worldvolume coupling

$$
S \supset \int_{\mathbb{R} P^{5}} F \cup C_{3}=\frac{1}{2} \int_{\mathbb{R} P^{3}} C_{3},
$$

to conclude that the D4-brane carries half a unit of D2 charge.

In general the obstruction to being spin is the second Stiefel-Whitney class $w_{2}$, and so $F$ is shifted by $w_{2}(N)$, yielding $w_{2}(N)$ units of $\mathrm{D} p$-brane charge inside of a $\mathrm{D}(p+2)$-brane, although we will soon see that the normalization is very subtle. We may calculate this obstruction directly from the cohomology of $\mathbb{R} P^{7}$. If we say that $a^{2}$ is the generator of $H^{2}\left(\mathbb{R} P^{7}\right)=\mathbb{Z}_{2}$, so that $a^{4}$ and $a^{6}$ generate $H^{4}$ and $H^{6}$, respectively, then the D4-brane is the Poincaré dual (PD) of $a^{2}$. To calculate the D2-brane charge corresponding to a $\mathrm{D} 4$-brane $\operatorname{PD}\left(a^{2}\right)$ we need to calculate $w_{2}$ of the normal bundle of $\operatorname{PD}\left(a^{2}\right)$, which gives the worldvolume field strength, and then push that forward onto the whole space so that we get the divisor of the corresponding $U(1)$ gauge bundle (the codimension two subset of our D4 that corresponds to the $F$-flux), which is our potential D2-brane. This operation is done by the cohomology operation $\mathrm{sq}^{2}$, and so we may argue that the D2-charge is given by

$$
\mathrm{PD}(\mathrm{D} 2) \stackrel{?}{=} \mathrm{sq}^{2} a^{2}=a^{4} .
$$

This is nonzero and so we see the D2-brane charge, however we have not been careful about the normalization. In fact the extension problem told us that the D4 should carry only half of a D2-brane.

This argument applied to the D0-brane charge in the D2-brane fails. The problem is that the D2-brane wraps $\mathbb{R} P^{3} \subset \mathbb{R} P^{7}$ whose normal bundle is the sum of the normal bundles of $\mathbb{R} P^{3} \subset \mathbb{R} P^{5}$ and $\mathbb{R} P^{5} \subset \mathbb{R} P^{7}$. While neither summand is spin, the $w_{2}$ 's of the summands cancel and so the normal 
bundle of the D2-brane is spin. It then seems as though the D2-brane should carry integral D0-charge. In terms of the $\mathrm{sq}^{2}$ construction we see the same unfortunate cancellation

$$
\mathrm{PD}(\mathrm{D} 0) \stackrel{?}{=} \mathrm{sq}^{2} \mathrm{sq}^{2} a^{2}=\mathrm{sq}^{2} a^{4}=\left(\mathrm{sq}^{2} a^{2}\right) a^{2}+a^{2}\left(\mathrm{sq}^{2} a^{2}\right)=a^{6}+a^{6}=2 a^{6}=0,
$$

where the last equality comes from the fact that $a^{6}$ generates the $\mathbb{Z}_{2}$ torsion group $H^{6}\left(\mathbb{R} P^{7}\right)$. The two $a^{6}$ 's that cancel are the two $w_{2}$ 's of the normal bundle summands. However the overall normalization of the worldvolume $\mathrm{D} p$ charge from the Wess-Zumino terms of the worldvolume action is a Chern character and so lives in rational cohomology and has no natural normalization $^{4}$ until we attempt to apply Dirac quantization. That is, the lift of the charge to an integral class and so the normalization of the charge should be derivable from the physics, and vice versa.

We know from the solution to the extension problem that the correct normalization yields the nontrivial D0 charge $a^{6}$ inside of our D2-brane or D4-brane. This is one half of the value found in equation (2.13). To see that this half is canonically defined we will need to include it before we make the transition from integral to rational cohomology, that is in the Steenrod square expression

$$
\mathrm{PD}(\mathrm{D} 0)=\frac{1}{2} \mathrm{sq}^{2} \mathrm{sq}^{2} a^{2}
$$

This is the same factor of $1 / 2$ that appeared in the formula for $d_{5}$ in Ref. [26]. As in that case, the division by two may be defined by rewriting the expression in terms of the Steenrod ${ }^{5}$ cube $P^{1}$, which cubes 2-cocyles modulo 3

$$
\mathrm{PD}(\mathrm{D} 0)=P^{1} \mathrm{PD}(\mathrm{D} 4)=P^{1} a^{2}=a^{6} .
$$

This agrees with the interpretation of branes that are not closed under the AHSS differentials $d_{2 k+1}$ as baryons. For example if $b$ is PD to a $\mathrm{D} p$-brane and

$$
d_{3} b=S q^{3} b=\beta \mathrm{sq}^{2} b=c \neq 0
$$

then the $\mathrm{D} p$-brane carries the charge of a half $\mathrm{D}(p-2)$-brane wrapped about $\mathrm{PD}\left(\mathrm{sq}^{2} b\right)$. However $\mathrm{PD}\left(\mathrm{sq}^{2} b\right)$ does not lift to integral homology because it is not in the kernel of $\beta$ and so the half $\mathrm{D}(p-2)$-brane, being oriented, has a boundary $(\mathrm{PD}(c))$ inside the worldvolume. This means that the half-brane must continue past this boundary into the bulk (wrapping $\mathrm{PD}(c)$ ) and so the $\mathrm{D} p$-brane is a baryon.

\footnotetext{
${ }^{4}$ It may be interesting to see how the inclusion of the $\sqrt{\hat{A}}$ term changes this situation.

${ }^{5}$ Acting on 3 -torsion $P^{1}$ is the usual cube, which yields the Milnor primitive term $Q_{1}=\beta P^{1}$ in $d_{5}$. However acting on 2 -torsion we define it to be a secondary operation that only acts on the cohomology of $d_{3}$.
} 
The extra factor of 2 allows us to extend this story to $d_{5}$ if

$$
d_{5} b=\beta P^{1} b=c \neq 0 .
$$

Generalizing equation (2.15) the $\mathrm{D} p$-brane dual to $b$ carries $P^{1} b$ units of $\mathrm{D}(p-4)$ charge. The units are smaller by a factor of two than those obtained by iterating the $d_{3}$ argument twice, but even this quarter $\mathrm{D}(p-4)$-brane has no boundary when $c \neq 0$ and so continues into the bulk, creating a baryon. The fact that $\mathrm{D} p$-branes not closed under $d_{5}$ yield $\mathrm{D} p$ - $\mathrm{D}(p-4)$ baryons was first noted in Ref. [33]. It should be possible to extend this reasoning to all of the differentials, such as the yet higher Steenrod powers that are relevant for string theory on $\mathbb{R} P^{9} \times S^{1}$. Including a $B$-field in this argument may lead to the construction of the twisted differentials as the Bockstein of the corresponding lower brane charges, which may be decomposed into $B$ terms and the lower-dimensional brane charges of the untwisted case following the strategy of Ref. [34].

\section{Computing the twisted $K$-theories of $\mathbb{R} P^{3} \times \mathbb{R} P^{7}$}

We are ultimately interested in the twisted $K$-theories of $\mathbb{R} P^{3} \times \mathbb{R} P^{2 k+1} \times$ $S^{6-2 k}(k=1,2,3)$. However, all of these may be obtained from the those of $\mathbb{R} P^{3} \times \mathbb{R} P^{7}$ by removing $3-k$ of the $\mathbb{Z}_{2}$ 's and using the Künneth formula to include the extra sphere. Thus in this section we will only compute the twisted $K$-theory of $\mathbb{R} P^{3} \times \mathbb{R} P^{7}$ and we will state the results for the other cases.

\subsection{Computing the cohomology of $\mathbb{R} P^{3} \times \mathbb{R} P^{7}$}

The real projective space $\mathbb{R} P^{n}$ is the quotient of the $n$-sphere $S^{n}$ by the antipodal map. The cohomology ring with $\mathbb{Z}_{2}$ coefficients is just a polynomial ring over $\mathbb{Z}_{2}$

$$
H^{*}\left(\mathbb{R} P^{n} ; \mathbb{Z}_{2}\right)=\mathbb{Z}_{2}[a] /\left(a^{n+1}\right),
$$

where $a$ is the 1-dimensional cocycle. Intuitively, $a^{k}$ is supported on the $\mathbb{R} P^{k} \subset \mathbb{R} P^{n}$. The $\mathbb{Z}$ homology and cohomology are only slightly more complicated. Again they are generated by the $\mathbb{R} P^{k}$ subsets, but with the boundary map

$$
\partial: C_{2 k} \longrightarrow C_{2 k-1}: a_{2 k} \longmapsto 2 a_{2 k-1},
$$

acting on the generator $a_{2 k}$ of the $2 k$-chains, intuitively the subset $\mathbb{R} P^{2 k}$. Dualizing, one obtains the coboundary map on the $(2 k-1)$-cochains

$$
\partial^{\dagger}: C^{2 k-1} \longrightarrow C^{2 k}: a^{2 k-1} \longmapsto 2 a^{2 k} \text {. }
$$


Thus the even cycles and odd cocycles are zero, while the odd cycles and even cocycles are boundaries and coboundaries if they are multiples of two. If $n$ is odd, as it will be in this example, then the top (and as always the bottom) dimensional chain and cochain are in the kernel and never the image of the differential, and so the top dimensional homology and cohomology is $\mathbb{Z}$.

We have then found, for the cases $n=3$ and $n=7$, that the nontrivial classes are

$$
\begin{aligned}
& H_{0}\left(\mathbb{R} P^{3}\right)=H_{0}\left(\mathbb{R} P^{7}\right)=H_{3}\left(\mathbb{R} P^{3}\right)=H_{7}\left(\mathbb{R} P^{7}\right)=\mathbb{Z}, \\
& H_{1}\left(\mathbb{R} P^{3}\right)=H_{1}\left(\mathbb{R} P^{7}\right)=H_{3}\left(\mathbb{R} P^{7}\right)=H_{5}\left(\mathbb{R} P^{7}\right)=\mathbb{Z}_{2}, \\
& H^{0}\left(\mathbb{R} P^{3}\right)=H^{0}\left(\mathbb{R} P^{7}\right)=H^{3}\left(\mathbb{R} P^{3}\right)=H^{7}\left(\mathbb{R} P^{7}\right)=\mathbb{Z} \\
& H^{2}\left(\mathbb{R} P^{3}\right)=H^{2}\left(\mathbb{R} P^{7}\right)=H^{4}\left(\mathbb{R} P^{7}\right)=H^{6}\left(\mathbb{R} P^{7}\right)=\mathbb{Z}_{2} .
\end{aligned}
$$

Our $\mathbb{Z}_{2}$ cohomology ring generator $a$ is not a cocycle now that we are using $\mathbb{Z}$ coefficients because it has coboundary $\partial^{\dagger} a=2 a^{2} \neq 0$. However we may still evaluate each term in the cohomology ring modulo two and so identify it with a subring of the $\mathbb{Z}_{2}$ cohomology. This allows us to write the $\mathbb{Z}$ cohomology generator of $H^{k}$ in terms of the $\mathbb{Z}_{2}$ generator $a$, basically $H^{k}$ is generated by $a^{k}$. Since we know how to multiply the $\mathbb{Z}_{2}$ generators (3.1) we can then guess how to multiply the $\mathbb{Z}$ generators.

We let $a$ and $b$ be the generators of $H^{1}\left(\mathbb{R} P^{3}, \mathbb{Z}_{2}\right)$ and $H^{1}\left(\mathbb{R} P^{7}, \mathbb{Z}_{2}\right)$, respectively. Although $a$ and $b$ themselves do not lift to integral classes, we will write the generators of the integral classes as powers of $a$ and $b$. Then we may encode the ring structure in the following definition, where $\langle x\rangle$ is the additive group generated by $x$

$$
\begin{gathered}
H^{0}\left(\mathbb{R} P^{3}\right)=\langle 1\rangle, \quad H^{2}\left(\mathbb{R} P^{3}\right)=\left\langle a^{2}\right\rangle, \quad H^{3}\left(\mathbb{R} P^{3}\right)=\left\langle a^{3}\right\rangle, \\
H^{0}\left(\mathbb{R} P^{7}\right)=\langle 1\rangle, \quad H^{2}\left(\mathbb{R} P^{7}\right)=\left\langle b^{2}\right\rangle, \quad H^{4}\left(\mathbb{R} P^{7}\right)=\left\langle b^{4}\right\rangle, \\
H^{6}\left(\mathbb{R} P^{7}\right)=\left\langle b^{6}\right\rangle, \quad H^{7}\left(\mathbb{R} P^{7}\right)=\left\langle b^{7}\right\rangle .
\end{gathered}
$$

We ultimately want the cohomology of the product. To find this, we will first evaluate the homology of the product using the Künneth formula

$$
\begin{aligned}
H_{n}\left(\mathbb{R} P^{3} \times \mathbb{R} P^{7}\right)= & \bigoplus_{i}\left(H_{i}\left(\mathbb{R} P^{3}\right) \otimes H_{n-i}\left(\mathbb{R} P^{7}\right)\right) \bigoplus \\
& \times\left(\bigoplus_{i} \operatorname{Tor}\left(H_{i}\left(\mathbb{R} P^{3}\right), H_{n-i-1}\left(\mathbb{R} P^{7}\right)\right)\right),
\end{aligned}
$$

where the only nontrivial Tor term will be

$$
\operatorname{Tor}\left(\mathbb{Z}_{2}, \mathbb{Z}_{2}\right)=\mathbb{Z}_{2}
$$


The Tor terms contribute a $\mathbb{Z}_{2}$ to $\mathrm{H}_{3}, \mathrm{H}_{5}$ and $\mathrm{H}_{7}$, the rest of the homology groups are just given by crossing homology classes of the components. In all we find

$$
H_{*}\left(\mathbb{R} P^{3} \times \mathbb{R} P^{7}\right)=\left(\mathbb{Z}, \mathbb{Z}_{2}^{2}, \mathbb{Z}_{2}, \mathbb{Z} \oplus \mathbb{Z}_{2}^{2}, \mathbb{Z}_{2}^{2}, \mathbb{Z}_{2}^{2}, \mathbb{Z}_{2}^{2}, \mathbb{Z} \oplus \mathbb{Z}_{2}, \mathbb{Z}_{2}^{2}, 0, \mathbb{Z}\right) .
$$

The universal coefficient theorem says that the cohomology groups are isomorphic to the homology groups but with the torsion parts moved up one dimension

$$
H^{*}\left(\mathbb{R} P^{3} \times \mathbb{R} P^{7}\right)=\left(\mathbb{Z}, 0, \mathbb{Z}_{2}^{2}, \mathbb{Z} \oplus \mathbb{Z}_{2}, \mathbb{Z}_{2}^{2}, \mathbb{Z}_{2}^{2}, \mathbb{Z}_{2}^{2}, \mathbb{Z} \oplus \mathbb{Z}_{2}^{2}, \mathbb{Z}_{2}, \mathbb{Z}_{2}^{2}, \mathbb{Z}\right)
$$

We are not interested in just the additive structure of $H^{*}$, but also the multiplicative structure. As in the case of $H^{*}\left(\mathbb{R} P^{7}\right)$ above, we can learn the multiplicative structure by writing the generators of $H^{*}\left(\mathbb{R} P^{3} \times \mathbb{R} P^{7}\right)$ in terms of the $\mathbb{Z}_{2}$ cohomology rings of the constituent real projective spaces. In most cases this will be made easier by the fact that the classes in the product cohomology are just products of the $\mathbb{Z}$ classes of the constituent cohomologies given in equation (3.1). The exceptions are the three classes $H^{3}, H^{5}$ and $H^{7}$ in which the Tor term contributed an extra $\mathbb{Z}_{2}$ to the homology. These cohomology classes will correspondingly contain an extra $\mathbb{Z}_{2}$ factor that is not a product of two cohomology classes of the components, but can be expressed as the Bockstein of a product of two $\mathbb{Z}_{2}$ cohomology classes $a$ and $b^{2 j+1}$ of the constituent $\mathbb{R} P^{n}$ 's (table 1 ).

\subsection{The untwisted $K$-theory of $\mathbb{R} P^{3} \times \mathbb{R} P^{7}$}

We will compute the (untwisted) $K$-theory of $\mathbb{R} P^{3} \times \mathbb{R} P^{7}$ using the AHSS. In fact only the first differential of this sequence

$$
d_{3}=S q^{3}
$$

will be nontrivial. $S q^{3}$ is the third Steenrod square. It can be decomposed as

$$
S q^{3}=\beta \mathrm{sq}^{2},
$$

where $\beta$ is the Bockstein map which lifts torsion $p$-cocycles to the $(p+1)$ st integral cohomology. The Bockstein map on $\mathbb{Z}_{2}$ classes has the following

Table 1: The cohomology groups and their generators are summarized.

\begin{tabular}{ccccccccccc}
\hline$H^{0}$ & $H^{1}$ & $H^{2}$ & $H^{3}$ & $H^{4}$ & $H^{5}$ & $H^{6}$ & $H^{7}$ & $H^{8}$ & $H^{9}$ & $H^{10}$ \\
\hline $\mathbb{Z}$ & 0 & $\mathbb{Z}_{2}^{2}$ & $\mathbb{Z} \oplus \mathbb{Z}_{2}$ & $\mathbb{Z}_{2}^{2}$ & $\mathbb{Z}_{2}^{2}$ & $\mathbb{Z}_{2}^{2}$ & $\mathbb{Z} \oplus \mathbb{Z}_{2}^{2}$ & $\mathbb{Z}_{2}$ & $\mathbb{Z}_{2}^{2}$ & $\mathbb{Z}$ \\
1 & & $a^{2}$ & $a^{3}$ & $b^{4}$ & $a^{3} b^{2}$ & $b^{6}$ & $b^{7}, a^{3} b^{4}$ & $a^{2} b^{6}$ & $a^{3} b^{6}$ & $a^{3} b^{7}$ \\
& & $b^{2}$ & $a b^{2}+a^{2} b$ & $a^{2} b^{2}$ & $a b^{4}+a^{2} b^{3}$ & $a^{2} b^{4}$ & $a b^{6}+a^{2} b^{5}$ & & $a^{2} b^{7}$ & \\
\hline
\end{tabular}


physical interpretation. Branes in type II string theory are necessarily oriented. Therefore if a half brane is wrapped on a nonorientable $(p+1)$-chain $Z_{p+1}$ then it must fail to close ${ }^{6}$ on the $p$-cycle $\partial Z_{p+1}$ which, due to the nonorientability of $Z_{p+1}$, is divisible by two. This means that, in addition to wrapping $Z_{p+1}$, it necessarily extends away from $Z_{p+1}$ making a cylinder whose cross-sections are each $\partial Z_{p+1}$. The Bockstein is dual to the half of $\partial Z_{p+1}$ that is wrapped by the cylindrical brane, and so the Bockstein map takes a half-brane to the cross-section of the tube of whole-brane that must be inserted to make it consistent (oriented). The half-brane itself will, in this case, result from the $\mathrm{sq}^{2}$ term.

As a first step we will compute $S q^{3}$ on all of the generators of our cohomology ring. Recall that all of the generators but three are products of integer classes of the cohomology of the individual $\mathbb{R} P^{n}$ 's. These integer classes are either zero classes or top classes, which are annihilated by $\mathrm{sq}^{2}$, or else products of even integral classes. If they are products of degree two integral classes then we may iteratively use the Cartan rule for Steenrod square two

$$
\mathrm{sq}^{2}(a b)=\left(\mathrm{sq}^{2} a\right) b+\left(S q^{1} a\right)\left(S q^{1} b\right)+a\left(\mathrm{sq}^{2} b\right),
$$

and the fact that $S q^{1}=\beta$ annihilates integral classes, to write $\mathrm{sq}^{2}$ on each class as an integral class times $\mathrm{sq}^{2}$ of an integral 2-class. $\mathrm{sq}^{2}$ acts on any 2-class by squaring it, and so it yields another integral class. Thus all of our generators except for possibly the three special ones will be mapped to integral classes $\left(\mathbb{Z}_{2}\right.$ classes with integral lifts) by $\mathrm{sq}^{2}$.

The Bockstein map annihilates $\mathbb{Z}_{2}$ classes that lift to integral classes of the same dimension. Thus $S q^{3}$ will annihilate all of our generators, except for possibly the three special ones. One of the special generators, $a b^{6}+a^{2} b^{5}$, is degree 7 and so $S q^{3}$ of it will be degree 10. The image of $S q^{3}$ is always $\bmod 2$ torsion, but $H^{10}\left(\mathbb{R} P^{3} \times \mathbb{R} P^{7}\right)$ does not contain any torsion, thus $S q^{3}$ will annihilate this generator as well. We then only need to evaluate $S q^{3}$ on the other two special generators.

We may evaluate $S q^{3}$ on the first special generator by using the fact that $S q^{3}$ squares three classes. Thus

$$
S q^{3}\left(a b^{2}+a^{2} b\right)=\left(a b^{2}+a^{2} b\right)\left(a b^{2}+a^{2} b\right)=a^{2} b^{4}+a^{4} b^{2}=a^{2} b^{4} .
$$

The second term vanishes because $a^{4}=0$. To evaluate $S q^{3}$ on the second generator we will also use the Cartan rule for $S q^{3}$ and the fact that all of

\footnotetext{
${ }^{6}$ Intuitively, the orientation of the brane may be defined everywhere except for a Dirac string, and that Dirac string is the insertion.
} 
the Steenrod squares annihilate powers of $a$ except for $S q^{1} a=a^{2}$.

$$
\begin{aligned}
S q^{3}\left(a b^{4}+a^{2} b^{3}\right)= & S q^{3}\left(a b^{4}\right)+S q^{3}\left(a^{2} b^{3}\right)=\left(S q^{1} a\right) \mathrm{sq}^{2} b^{4}+a S q^{3} b^{4}+a^{2} S q^{3} b^{3} \\
= & \left(a^{2}\right)\left[\left(\mathrm{sq}^{2} b^{2}\right) b^{2}+b^{2}\left(\mathrm{sq}^{2} b^{2}\right)\right]+a \beta\left[\left(\mathrm{sq}^{2} b^{2}\right) b^{2}\right. \\
& \left.+b^{2}\left(\mathrm{sq}^{2} b^{2}\right)\right]+a^{2} b^{6} \\
= & a^{2}\left[2 b^{6}\right]+a \beta\left[2 b^{6}\right]+a^{2} b^{6}=0+0+a^{2} b^{6}=a^{2} b^{6} .
\end{aligned}
$$

We may now use the AHSS to compute the associated graded part of the untwisted $K$-theory of $\mathbb{R} P^{3} \times \mathbb{R} P^{7}$. It is just given by the cohomology of $S q^{3}$

$$
\begin{aligned}
& \operatorname{Gr}\left(K^{0}\right)=\frac{\operatorname{Ker}\left(S q^{3}\right): H^{\text {even }} \longrightarrow H^{\text {odd }}}{\operatorname{Im}\left(S q^{3}\right): H^{\text {odd }} \longrightarrow H^{\text {even }}}=\frac{\mathbb{Z}^{2} \oplus \mathbb{Z}_{2}^{7}}{\mathbb{Z}_{2}^{2}}=\mathbb{Z}^{2} \oplus \mathbb{Z}_{2}^{5}, \\
& \operatorname{Gr}\left(K^{1}\right)=\frac{\operatorname{Ker}\left(S q^{3}\right): H^{\text {odd }} \longrightarrow H^{\text {even }}}{\operatorname{Im}\left(S q^{3}\right): H^{\text {even }} \longrightarrow H^{\text {odd }}}=\frac{\mathbb{Z}^{2} \oplus \mathbb{Z}_{2}^{5}}{0}=\mathbb{Z}^{2} \oplus \mathbb{Z}_{2}^{5}
\end{aligned}
$$

To calculate the $K$-theory ring from the associated graded $K$-group one needs to solve an extension problem, which in this case allows each $\mathbb{Z}$ to eat either zero or some $\mathbb{Z}_{2}$ 's and allows the $\mathbb{Z}_{2}$ 's to combine into $\mathbb{Z}_{2^{j}}$ 's. We may alternatively find the $K$-theory by $T$-dualizing the configuration to $S^{2} \times \mathbb{C} P^{3} \times T^{2}$ with $H$-flux, explicitly constructing the bundles on $\mathbb{C} P^{3}$, and noting that two vortices make an instanton while two instantons make a codimension 6 instanton, which corresponds to the assembly of the three corresponding $\mathbb{Z}_{2}$ 's into a $\mathbb{Z}_{8}$.

Instead, in the spirit of Ref. [28], we will solve for the untwisted $K$-theory of $\mathbb{R} P^{3} \times \mathbb{R} P^{7}$ by using the Künneth formula for $K$-homology

$$
0 \longrightarrow K_{*}(A) \otimes K_{*}(B) \longrightarrow K_{*}(A \times B) \longrightarrow \operatorname{Tor}\left(K_{*}(A), K_{*}(B)\right) \longrightarrow 0
$$

as well as the untwisted $K$-homologies of the constituents

$$
\begin{array}{ll}
K_{0}\left(\mathbb{R} P^{3}\right)=\mathbb{Z}, \quad K_{1}\left(\mathbb{R} P^{3}\right)=\mathbb{Z} \oplus \mathbb{Z}_{2}, \\
K_{0}\left(\mathbb{R} P^{7}\right)=\mathbb{Z}, \quad K_{1}\left(\mathbb{R} P^{7}\right)=\mathbb{Z} \oplus \mathbb{Z}_{8} .
\end{array}
$$

Using the fact that $\mathbb{Z}_{2} \otimes \mathbb{Z}_{8}=\mathbb{Z}_{2}$ and that Tor $(x, y)$ vanishes unless both $x$ and $y$ contain torsion components we find that

$$
\begin{aligned}
K_{0}\left(\mathbb{R} P^{3} \times \mathbb{R} P^{7}\right)= & \left(K_{0}\left(\mathbb{R} P^{3}\right) \otimes K_{0}\left(\mathbb{R} P^{7}\right)\right) \oplus\left(K_{1}\left(\mathbb{R} P^{3}\right) \otimes K_{1}\left(\mathbb{R} P^{7}\right)\right) \\
& \oplus \operatorname{Tor}\left(K_{0}\left(\mathbb{R} P^{3}\right), K_{1}\left(\mathbb{R} P^{7}\right)\right) \oplus \operatorname{Tor}\left(K_{1}\left(\mathbb{R} P^{3}\right), K_{0}\left(\mathbb{R} P^{7}\right)\right) \\
= & (\mathbb{Z} \otimes \mathbb{Z}) \oplus\left(\left(\mathbb{Z} \oplus \mathbb{Z}_{2}\right) \otimes\left(\mathbb{Z} \oplus \mathbb{Z}_{8}\right)\right) \oplus 0 \oplus 0 \\
= & \mathbb{Z}^{2} \oplus \mathbb{Z}_{8} \oplus \mathbb{Z}_{2}^{2} .
\end{aligned}
$$


while $\operatorname{Tor}\left(\mathbb{Z}_{2}, \mathbb{Z}_{8}\right)=\mathbb{Z}_{2}$ yields

$$
\begin{aligned}
K_{1}\left(\mathbb{R} P^{3} \times \mathbb{R} P^{7}\right)= & \left(K_{0}\left(\mathbb{R} P^{3}\right) \otimes K_{1}\left(\mathbb{R} P^{7}\right)\right) \oplus\left(K_{1}\left(\mathbb{R} P^{3}\right) \otimes K_{0}\left(\mathbb{R} P^{7}\right)\right) \\
& \oplus \operatorname{Tor}\left(K_{0}\left(\mathbb{R} P^{3}\right), K_{0}\left(\mathbb{R} P^{7}\right)\right) \oplus \operatorname{Tor}\left(K_{1}\left(\mathbb{R} P^{3}\right), K_{1}\left(\mathbb{R} P^{7}\right)\right) \\
= & \left(\mathbb{Z} \otimes\left(\mathbb{Z} \oplus \mathbb{Z}_{8}\right)\right) \oplus\left(\left(\mathbb{Z} \oplus \mathbb{Z}_{2}\right) \otimes \mathbb{Z}\right) \oplus 0 \oplus \operatorname{Tor}\left(\mathbb{Z}_{2}, \mathbb{Z}_{8}\right) \\
= & \mathbb{Z}^{2} \oplus \mathbb{Z}_{8} \oplus \mathbb{Z}_{2}^{2} .
\end{aligned}
$$

The universal coefficient theorem then yields the desired $K$-cohomology groups

$$
K^{0}\left(\mathbb{R} P^{3} \times \mathbb{R} P^{7}\right)=\mathbb{Z}^{2} \oplus \mathbb{Z}_{8} \oplus \mathbb{Z}_{2}^{2}, \quad K^{1}\left(\mathbb{R} P^{3} \times \mathbb{R} P^{7}\right)=\mathbb{Z}^{2} \oplus \mathbb{Z}_{8} \oplus \mathbb{Z}_{2}^{2} .
$$

These $K$-theory classes yield flux backgrounds or brane configurations for type IIA or IIB string theory. In IIB string theory the $K^{1}$ classes describe possible RR fields in the absence of an NS flux, and if we $S$-dualize a configuration in which the $G_{3}$-flux valued in $\mathbb{Z} \subset H^{3}\left(\mathbb{R} P^{3} \times \mathbb{R} P^{7}\right)$ is nonzero then we find another configuration which corresponds to a class in the $K$-theory twisted by the original $G_{3}$.

The $K$-homology groups are also both $\mathbb{Z}^{2} \oplus \mathbb{Z}_{8} \oplus \mathbb{Z}_{2}^{2}$. In type IIB the $\mathbb{Z}$ 's are generated by the $D(-1)$ and the D9. The $\mathbb{Z}_{8}$ is generated by a D7 wrapping $\mathbb{R} P^{3} \times \mathbb{R} P^{5}$. Similarly to the case of Section 2 , the element $2 \in \mathbb{Z}_{8}$ corresponds to a D5 wrapping $\mathbb{R} P^{3} \times \mathbb{R} P^{3}$ and 4 to a D3 wrapping $\mathbb{R} P^{3} \times \mathbb{R} P^{1}$. The $\mathbb{Z}_{2}$ generators are the D7 wrapping $\mathbb{R} P^{1} \times \mathbb{R} P^{7}$ and the D5 wrapping $\mathbb{R} P^{1} \times \mathbb{R} P^{5}$. One might be tempted to think that the D5 wrapping $\mathbb{R} P^{1} \times \mathbb{R} P^{5}$ would be $\mathbb{Z}_{8}$ charged like its cousin that wraps $\mathbb{R} P^{3} \times \mathbb{R} P^{5}$. Above we have seen that the $\mathbb{Z}_{2}$ charge results from the fact that $\mathbb{Z}_{2} \otimes \mathbb{Z}_{8}=$ $\mathbb{Z}_{2}$. Physically this is a consequence of the instability of the element 2 , which is the D3 wrapping $\mathbb{R} P^{1} \times \mathbb{R} P^{3}$. The D3 may split into two D5's which each wrap $\mathbb{R} P^{1} \times \mathbb{R} P^{5}$ and carry a half unit of D3 charge. These two D5's each wrap the circle $\mathbb{R} P^{1} \subset \mathbb{R} P^{3}$ and they may connect so as to wrap the circle $\mathbb{R} P^{1} \subset \mathbb{R} P^{3}$ twice. However twice this circle is the boundary of a $\mathbb{R} P^{2} \subset \mathbb{R} P^{3}$, that is $\partial \mathbb{R} P^{2}=2 \mathbb{R} P^{1}$. Thus the doubly-wrapped D5 may decay by sweeping out $\mathbb{R} P^{2}$. This entire process is mathematically just the distributive property of the tensor product

$$
\left(1 \in \mathbb{Z}_{2}\right) \otimes\left(2 \in \mathbb{Z}_{8}\right)=\left(2 \in \mathbb{Z}_{2}\right) \otimes\left(1 \in \mathbb{Z}_{8}\right)=0 \otimes\left(1 \in \mathbb{Z}_{8}\right)=0 .
$$

We summarize the wrappings and the corresponding brane charges as elements of $\mathbb{Z} \oplus \mathbb{Z} \oplus \mathbb{Z}_{8} \oplus \mathbb{Z}_{2} \oplus \mathbb{Z}_{2}$ in the following table, where the top row is the element of $K_{0}$ and the bottom row is the subset of $\mathbb{R} P^{3} \times \mathbb{R} P^{7}$ wrapped (table 2).

Again one may replace $\mathbb{R} P^{7}$ by $\mathbb{R} P^{2 k+1}$ by replacing the $\mathbb{Z}_{8}$ 's by $Z_{2^{k}}$ 's, as there will be only $k \mathbb{Z}_{2}$ 's. The terms with $b^{j>2 k+1}$ are no longer present as 
Table 2: Some $K$-homology classes and the corresponding wrapped cycles.

\begin{tabular}{lllllll}
\hline$\left(1,0^{4}\right)$ & $\left(0,1,0^{3}\right)$ & $\left(0^{2}, 1,0^{2}\right)$ & $\left(0^{2}, 2,0^{2}\right)$ & $\left(0^{2}, 4,0^{2}\right)$ & $\left(0^{3}, 1,0\right)$ & $\left(0^{4}, 1\right)$ \\
\hline
\end{tabular}

a point $\mathbb{R} P^{3} \times \mathbb{R} P^{7} \mathbb{R} P^{3} \times \mathbb{R} P^{5} \mathbb{R} P^{3} \times \mathbb{R} P^{3} \mathbb{R} P^{3} \times \mathbb{R} P^{1} \mathbb{R} P^{1} \times \mathbb{R} P^{7} \mathbb{R} P^{1} \times \mathbb{R} P^{5}$

they do not fit in $\mathbb{R} P^{2 k+1}$. Crossing the spacetime by an even-dimensional sphere just corresponds to doubling the $K$-groups $K \mapsto K \oplus K$.

\subsection{The twisted $K$-theory}

We are interested in configurations that do not correspond to $S$-covariant classes. To construct such a configuration we turn on an $H$-flux valued in the $\mathbb{Z}_{2}$ part of $H^{3}\left(\mathbb{R} P^{3} \times \mathbb{R} P^{2 k+1} \times S^{6-2 k}\right)$, that is

$$
H=a b^{2}+b a^{2} .
$$

If $k>1$ (so that $b^{4} \neq 0$ ) this will not yield an $S$-covariant configuration because, as we have just seen in equation (3.13),

$$
S q^{3} H=a^{2} b^{4} \neq 0 .
$$

However a twisted $K$-theory exists with every possible twist in $H^{3}$ with $\mathbb{Z}$ coefficients and so it does make sense to calculate the twisted $K$-theory $K_{H}\left(\mathbb{R} P^{3} \times \mathbb{R} P^{7}\right)$, so long as we remember that fluxes valued in this group will lead to anomalies that need to be canceled. If $k=3$ then

$$
H \cup H \cup H=a^{3} b^{6} \neq 0,
$$

which, as was argued in the introduction, implies that unless $H \cup P=$ $H \cup H \cup H$ the anomaly cannot be canceled and so the $k=3$ case will be unphysical. We have not been able to calculate $P$, however if indeed $P$ does contain a $a^{2} b^{4}$ term and so render the $k=3$ case consistent then the D3brane dual to $a^{2} b^{4}$ will not appear in the twisted case but instead will appear in the untwisted case. T-dualizing the untwisted case, as we will see in the next section, we arrive at a spacetime with no torsion and so the $T$-dual of this gravitational D3-brane should be a D-string produced by a FreedWitten anomaly of the fluxes. One might hope to compute $P$ by examining the Freed-Witten anomalies of these dual fluxes, but the result is heavily dependent on factors of two that we have so far been unable to determine. A D-string analogue of our proposed formula for D3-brane charge would be helpful. 
The associated graded twisted $K$-theory is in this case just the cohomology with respect to the differential

$$
d_{3}=S q^{3}+H
$$

and so we need to calculate the cup product of $H$ with all of our cohomology generators. Like $S q^{3}, H \cup$ will lead to a torsion class three degrees higher and so $H$ will automatically annihilate all of the classes of degree seven and above. Similarly $H$ will annihilate any term containing $a^{3}$, as $a^{3}$ is already of the maximum degree in $H^{*}\left(\mathbb{R} P^{3}\right)$. The products with $H$ are computed using multiplication in the respective $\mathbb{Z}_{2}$ rings and then lifting the results to integral cohomology. We find

$$
\begin{gathered}
H \cup 1=a b^{2}+a^{2} b, \quad H \cup a^{2}=a^{3} b^{2}, \quad H \cup b^{2}=a b^{4}+a^{2} b^{3}, \\
H \cup\left(a b^{2}+a^{2} b\right)=a^{2} b^{4}, \quad H \cup a^{2} b^{2}=a^{3} b^{4}, \quad H \cup b^{4}=a b^{6}+a^{2} b^{5}, \\
H \cup\left(a b^{4}+a^{2} b^{3}\right)=a^{2} b^{6} \quad H \cup a^{2} b^{4}=a^{3} b^{6} \quad H \cup b^{6}=a^{2} b^{7} .
\end{gathered}
$$

Note that while $S q^{3}$ and $H$ are both nontrivial on the special 3 and 5 cocycles $H$ and $H \cup b^{2}, d_{3}$ annihilates them both. However they are both in the image of $d_{3}$ and so they will be quotiented out of the final answer.

We may now assemble the above results to find the associated graded twisted $K$-theory.

$$
\begin{aligned}
\operatorname{Gr}\left(K_{H}^{0}\right) & =\frac{\operatorname{Ker}\left(d_{3}\right): H^{\text {even }} \longrightarrow H^{\text {odd }}}{\operatorname{Im}\left(d_{3}\right): H^{\text {odd }} \longrightarrow H^{\text {even }}}=\frac{\left\langle 2, a^{2} b^{6}, a^{3} b^{7}\right\rangle}{0}=\mathbb{Z}^{2} \oplus \mathbb{Z}_{2} \\
\operatorname{Gr}\left(K_{H}^{1}\right) & =\frac{\operatorname{Ker}\left(d_{3}\right): H^{\text {odd }} \longrightarrow H^{\text {even }}}{\operatorname{Im}\left(d_{3}\right): H^{\text {even }} \longrightarrow H^{\text {odd }}} \\
& =\frac{\left\langle a^{3}, b^{7}, a b^{2}+a^{2} b, a b^{4}+a^{2} b^{3}, a^{3} b^{2}, a^{3} b^{4}, a b^{6}+a^{2} b^{5}, a^{3} b^{6}, a^{2} b^{7}\right\rangle}{\left\langle a b^{2}+a^{2} b, a b^{4}+a^{2} b^{3}, a^{3} b^{2}, a^{3} b^{4}, a b^{6}+a^{2} b^{5}, a^{3} b^{6}, a^{2} b^{7}\right\rangle} \\
& =\frac{\mathbb{Z}^{2} \oplus \mathbb{Z}_{2}^{7}}{\mathbb{Z}_{2}^{7}}=\mathbb{Z}^{2} .
\end{aligned}
$$

$\operatorname{Gr}\left(K_{H}^{1}\right)$ has no torsion and so the extension problem for $K_{H}^{1}$ is trivial and we may conclude that

$$
K_{H}^{1}\left(\mathbb{R} P^{3} \times \mathbb{R} P^{7}\right)=\mathbb{Z}^{2} .
$$

However to find $K_{H}^{0}$ we need to solve the extension problem

$$
\mathbb{Z}^{2} \stackrel{f}{\longrightarrow} K_{H}^{0}\left(\mathbb{R} P^{3} \times \mathbb{R} P^{7}\right) \stackrel{g}{\longrightarrow} \mathbb{Z}_{2} .
$$

This admits two possible solutions. If the first map, $f$, were multiplication by one then the sequence would split and $K_{H}^{0}$ would be $\mathbb{Z}^{2} \oplus \mathbb{Z}_{2}$. If on the other hand $f$ were multiplication by two on one $\mathbb{Z}$ then $K_{H}^{0}$ would be $\mathbb{Z}^{2}$. A very similar extension problem occurs in the computation of the twisted 
$K$-theory of $\mathbb{R} P^{2 k+1} \times S^{1}$. There it has been shown by $T$-duality [23] that the map is indeed multiplication by two. We will argue in the next section that the configuration $\mathbb{R} P^{3} \times \mathbb{R} P^{7}$ with $H$-flux (3.23) is $T$-dual to $S^{3} \times S^{7}$ with no $H$-flux, for which the two $K$-theory groups are both $\mathbb{Z}^{2}$. Thus the fact that $T$-duality is an isomorphism of twisted $K$-theory $[23,35]$ will allow us to conclude that this time, as in the case of $\mathbb{R} P^{2 k+1} \times S^{1}$, $f$ is multiplication by two and so

$$
K_{H}^{0}\left(\mathbb{R} P^{3} \times \mathbb{R} P^{7}\right)=\mathbb{Z}^{2} .
$$

In Section 6, when we explicitly $T$-dualize the brane dual to the $\mathbb{Z}_{2}$ cycle $a^{2} b^{6}$, we will give a physical interpretation of the fact that $f$ is degree two.

The torsion has all been killed and so the twisted $K$-groups are $k$-independent. However note that all $K$-groups double to $\mathbb{Z}^{4}$ when we cross our $\mathbb{R} P^{3} \times \mathbb{R} P^{2 k+1}$ with a sphere.

\section{T-duality}

\subsection{The untwisted case: $\mathbb{C} P^{1} \times \mathbb{C} P^{3} \times T^{2}$}

As a warm up for the more difficult $T$-duality to come, we dualize the $k=3$ example of $\mathbb{R} P^{3} \times \mathbb{R} P^{7}$ with no $H$-flux. Both $\mathbb{R} P^{3}$ and $\mathbb{R} P^{7}$ admit free circle actions and in particular are circle bundles over the complex projective spaces $\mathbb{C} P^{1}$ and $\mathbb{C} P^{3}$ respectively

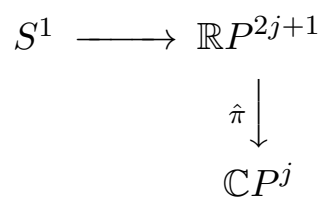

The nontrivial cohomology classes and generators of the base spaces are

$$
\begin{aligned}
H^{0}\left(\mathbb{C} P^{1}\right)=H^{0}\left(\mathbb{C} P^{3}\right)=\mathbb{Z} & =\langle 1\rangle, \quad H^{2}\left(\mathbb{C} P^{1}\right)=\mathbb{Z}=\langle\alpha\rangle, \\
H^{2}\left(\mathbb{C} P^{3}\right)=\mathbb{Z}=\langle\beta\rangle, \quad H^{4}\left(\mathbb{C} P^{3}\right) & =\mathbb{Z}=\left\langle\beta^{2}\right\rangle, \quad H^{6}\left(\mathbb{C} P^{3}\right)=\mathbb{Z}=\left\langle\beta^{3}\right\rangle,
\end{aligned}
$$

and the Chern classes of the two bundles are

$$
c_{1}=2 \alpha \quad \text { and } \quad c_{1}=2 \beta .
$$

We may now use the prescription of Ref. [23] to $T$-dualize both circle fibers. According to that prescription $T$-duality exchanges the integrals 
of the $H$-fluxes over the circle fibers with the Chern classes. The original $H$-flux vanishes and so the dual Chern classes vanish

$$
\hat{c}_{1}=0 \text {. }
$$

The dual spacetime $\widehat{M}$ then consists of the product of two trivial circle bundles over the original base

$$
\widehat{M}=\mathbb{C} P^{1} \times \mathbb{C} P^{3} \times S_{\alpha}^{1} \times S_{\beta}^{1} .
$$

The dual $H$-flux is just the sum of two $H$-fluxes that integrate to the Chern classes

$$
\widehat{H}=2 \alpha \cup \theta_{\alpha}+2 \beta \cup \theta_{\beta},
$$

where $\theta_{\alpha}$ and $\theta_{\beta}$ generate $H^{1}\left(S^{1}, \mathbb{Z}\right)$ of the dual circles.

We will now compute the twisted $K$-theory of $\widehat{M}$ which, as we have performed an even number of $T$-dualities, must agree with the untwisted $K$ theory of the original space given in equation (3.21). The cohomology of $\widehat{M}$ follows from the Künneth theorem, where the Tor terms all vanish as there is no torsion (table 3). The fact that the cohomology contains no torsion also implies that $S q^{3}$ annihilates everything. The cup product with $H$ acts as follows

$$
\begin{aligned}
& 1 \stackrel{H}{\longrightarrow} 2 \alpha \theta_{\alpha}+2 \beta \theta_{\beta}, \quad \theta_{\alpha} \stackrel{H}{\longrightarrow}-2 \beta \theta_{\alpha} \theta_{\beta}, \quad \alpha \stackrel{H}{\longrightarrow} 2 \alpha \beta \theta_{\beta}, \\
& \quad \alpha \theta_{\alpha} \stackrel{H}{\longrightarrow}-2 \alpha \beta \theta_{\alpha} \theta_{\beta}, \\
& \beta \stackrel{H}{\longrightarrow} 2 \alpha \beta \theta_{\alpha}+2 \beta^{2} \theta_{\beta}, \quad \beta \theta_{\alpha} \stackrel{H}{\longrightarrow}-2 \beta^{2} \theta_{\alpha} \theta_{\beta}, \quad \alpha \beta \stackrel{H}{\longrightarrow} 2 \alpha \beta^{2} \theta_{\beta}, \\
& \quad \alpha \beta \theta_{\alpha} \stackrel{H}{\longrightarrow}-2 \alpha \beta^{2} \theta_{\alpha} \theta_{\beta}, \\
& \beta^{2} \stackrel{H}{\longrightarrow} 2 \alpha \beta^{2} \theta_{\alpha}+2 \beta^{3} \theta_{\beta}, \quad \beta^{2} \theta_{\alpha} \stackrel{H}{\longrightarrow}-2 \beta^{3} \theta_{\alpha} \theta_{\beta}, \quad \alpha \beta^{2} \stackrel{H}{\longrightarrow} 2 \alpha \beta^{3} \theta_{\beta}, \\
& \quad \alpha \beta^{2} \theta_{\alpha} \stackrel{H}{\longrightarrow}-2 \alpha \beta^{3} \theta_{\alpha} \theta_{\beta}, \\
& \beta^{3} \stackrel{H}{\longrightarrow} 2 \alpha \beta^{3} \theta_{\alpha}, \quad \theta_{\beta} \stackrel{H}{\longrightarrow} 2 \alpha \theta_{\alpha} \theta_{\beta}, \quad \beta \theta_{\beta} \stackrel{H}{\longrightarrow} 2 \alpha \beta \theta_{\alpha} \theta_{\beta}, \\
& \quad \beta^{2} \theta_{\beta} \stackrel{H}{\longrightarrow} 2 \alpha \beta^{2} \theta_{\alpha} \theta_{\beta}, \quad \beta^{3} \theta_{\beta} \stackrel{H}{\longrightarrow} 2 \alpha \beta^{3} \theta_{\alpha} \theta_{\beta}, \\
& \alpha \theta_{\beta}, \alpha \beta \theta_{\beta}, \alpha \beta^{2} \theta_{\beta}, \alpha \beta^{3} \theta_{\beta}, \alpha \theta_{\alpha}+\beta \theta_{\beta}, \alpha \beta \theta_{\alpha}+\beta^{2} \theta_{\beta}, \alpha \beta^{2} \theta_{\alpha}+\beta^{3} \theta_{\beta}, \\
& \quad \alpha \beta^{3} \theta_{\alpha}, \beta^{3} \theta_{\alpha} \stackrel{H}{\longrightarrow} 0, \\
& \alpha \beta^{3}, \theta_{\alpha} \theta_{\beta}, \beta \theta_{\alpha} \theta_{\beta}, \beta^{2} \theta_{\alpha} \theta_{\beta}, \beta^{3} \theta_{\alpha} \theta_{\beta}, \alpha \theta_{\alpha} \theta_{\beta}, \alpha \beta \theta_{\alpha} \theta_{\beta}, \alpha \beta^{2} \theta_{\alpha} \theta_{\beta}, \alpha \beta^{3} \theta_{\alpha} \theta_{\beta} \stackrel{H}{\longrightarrow} 0 .
\end{aligned}
$$

The first approximation to $K^{i}$, which we will call $E_{1}^{i}$, is the even or odd part of the quotient of the kernel of $d_{3}=H \cup$ by the image of $d_{3}=H \cup$. 
Table 3: The cohomology groups of $\widehat{M}$ and their generators are summarized.

\begin{tabular}{ccccccccccc}
\hline$H^{0}$ & $H^{1}$ & $H^{2}$ & $H^{3}$ & $H^{4}$ & $H^{5}$ & $H^{6}$ & $H^{7}$ & $H^{8}$ & $H^{9}$ & $H^{10}$ \\
\hline $\mathbb{Z}$ & $\mathbb{Z}^{2}$ & $\mathbb{Z}^{3}$ & $\mathbb{Z}^{4}$ & $\mathbb{Z}^{4}$ & $\mathbb{Z}^{4}$ & $\mathbb{Z}^{4}$ & $\mathbb{Z}^{4}$ & $\mathbb{Z}^{3}$ & $\mathbb{Z}^{2}$ & $\mathbb{Z}$ \\
1 & $\theta_{\alpha}$ & $\alpha$ & $\alpha \theta_{\alpha}$ & $\alpha \theta_{\alpha} \theta_{\beta}$ & $\alpha \beta \theta_{\alpha}$ & $\alpha \beta \theta_{\alpha} \theta_{\beta}$ & $\alpha \beta^{2} \theta_{\alpha}$ & $\alpha \beta^{2} \theta_{\alpha} \theta_{\beta}$ & $\alpha \beta^{3} \theta_{\alpha}$ & $\alpha \beta^{3} \theta_{\alpha} \theta_{\beta}$ \\
& $\theta_{\beta}$ & $\beta$ & $\alpha \theta_{\beta}$ & $\alpha \beta$ & $\alpha \beta \theta_{\beta}$ & $\alpha \beta^{2}$ & $\alpha \beta^{2} \theta_{\beta}$ & $\alpha \beta^{3}$ & $\alpha \beta^{3} \theta_{\beta}$ & \\
& & $\theta_{\alpha} \theta_{\beta}$ & $\beta \theta_{\alpha}$ & $\beta \theta_{\alpha} \theta_{\beta}$ & $\beta^{2} \theta_{\alpha}$ & $\beta^{2} \theta_{\alpha} \theta_{\beta}$ & $\beta^{3} \theta_{\alpha}$ & $\beta^{3} \theta_{\alpha} \theta_{\beta}$ & & \\
& & & $\beta \theta_{\beta}$ & $\beta^{2}$ & $\beta^{2} \theta_{\beta}$ & $\beta^{3}$ & $\beta^{3} \theta_{\beta}$ & & & \\
\hline
\end{tabular}

Inspecting the above action of $H$ we see that

$$
E_{1}^{0}=E_{1}^{1}=\mathbb{Z}^{2} \times \mathbb{Z}_{2}^{7}
$$

These have bigger torsion subgroups, each with 128 elements, than the $T$ dual $K$-classes, which each had only 32 . While the extension problem could in principle resolve this discrepancy, we will argue that instead the extra classes are removed by the higher AHSS differential $d_{5}$. These will remove precisely the same elements that were removed, in the derivation of the $T$-dual $K$-theory using the Künneth theorem, by the fact that

$$
\mathbb{Z}_{8} \otimes \mathbb{Z}_{2}=\operatorname{Tor}\left(\mathbb{Z}_{8}, \mathbb{Z}_{2}\right)=\mathbb{Z}_{2},
$$

which is smaller than the original $\mathbb{Z}_{8}$ by a factor of 4 [28].

To find the action of $d_{5}$ we will use the dual description of these cohomology classes in terms of D-branes. The product of two $T$-dualities is an isomorphism of twisted $K$-theory and furthermore the extension problem is the same as before, yielding the same pattern of fractional brane charges inside of higher dimensional branes. In particular, the branes wrapping the $\mathbb{C} P^{2} \subset \mathbb{C} P^{3}$ each carry half a unit of charge of a brane wrapping $\mathbb{C} P^{1} \subset \mathbb{C} P^{3}$ while those wrapping $\mathbb{C} P^{1} \subset \mathbb{C} P^{3}$ each carry half a unit of charge of a brane at a point in the $\mathbb{C} P^{3}$ directions. The first of these facts follows from the fact that the normal bundle of $\mathbb{C} P^{2} \subset \mathbb{C} P^{3}$ is not spin, and the second follows from this argument with the same factor of 2 that we saw in the $K$-theory of $\mathbb{R} P^{7}$. In terms of the Steenrod algebra these two relations are consequences of

$$
\operatorname{sq}^{2} \beta=\beta^{2}, \quad P^{1} \beta=\beta^{3},
$$

where again $P^{1}$ acting on 2-torsion is a secondary operation that cubes 2-classes. In fact the $\mathbb{R} P^{7}$ case is just a pullback of this one using the projection map $\pi: \mathbb{R} P^{7} \longrightarrow \mathbb{C} P^{3}$ of equation (4.1).

Consider for example the D5-brane dual to $\alpha \theta_{\alpha}+\beta \theta_{\beta}$. This brane consists of two components, one wrapping $\mathbb{C} P^{3} \times S_{\beta}^{1}$ and the other wrapping $\mathbb{C} P^{1} \times$ $\mathbb{C} P^{2} \times S_{\alpha}^{1}$, where the extra dimension again comes from the fact that dual branes live in the space augmented by an extra deformation direction. Both 
of these components contain $H$-flux, but the orientations disagree and the total integral of $H$ cancels

$$
\int_{\mathbb{C} P^{3} \times S_{\beta}^{1}} H=-\int_{\mathbb{C} P^{1} \times \mathbb{C} P^{2} \times S_{\alpha}^{1}} H=2 \int_{\mathbb{C} P^{2}}
$$

Here the integral denotes the homology-cohomology pairing over the integers, and the terms are to be interpreted as operators that act on cohomology over the integers.

The $H$-flux on each component is nonvanishing, and so each component requires a D3-brane insertion to cancel the corresponding Freed-Witten anomaly, or equivalently, as a sink for the worldvolume magnetic flux sourced by the $H$-flux. The cancellation (4.11) means that the same number of D3branes are emitted from one D5 as are absorbed by the other and so this is not a D5-D3 baryon, reflecting the fact that $a \theta_{\alpha}+b \theta_{\beta}$ is $d_{3}$-closed. As there are two units of $H$-flux, two D3-branes connect the pair of D5's. To sink the magnetic flux sourced by $H$, the intersection of the pair of D3's and each D5 component needs to be PD to the $H$-flux restricted to the components worldvolume. This means that each D3 wraps $\mathbb{C} P^{2} \subset \mathbb{C} P^{3} \times S_{\beta}^{1}$ in the first component and $\mathbb{C} P^{2} \subset \mathbb{C} P^{1} \times \mathbb{C} P^{2} \times S_{\alpha}^{1}$ in the second. It is a critical test of the consistency of this picture that the topology of the tube of D3-brane is the same at both endpoints (figure 3).

As the normal bundle of $\mathbb{C} P^{2} \subset \mathbb{C} P^{3}$ is not spin, the D5 component wrapping $\mathbb{C} P^{1} \times \mathbb{C} P^{2} \times S_{\alpha}^{1}$ must contain a half unit of charge of a D3-brane wrapped on $\mathbb{C} P^{1} \times \mathbb{C} P^{1} \times S_{\alpha}^{1}$, which is dual to $w_{2}$ of its normal bundle. Similarly the D3-brane tube wraps $\mathbb{C} P^{2} \subset \mathbb{C} P^{3}$ times an interval and so it must contain a half unit of D1 charge on $\mathbb{C} P^{1} \subset \mathbb{C} P^{3}$ times the interval. On the other hand the second component $\mathbb{C} P^{3} \times S_{\beta}^{1}$, which has a trivial normal bundle, apparently supports no D3 charge. Thus the D1-charge inside of the D3-brane cannot end on the second component, and must instead continue off to infinity. There are two D3 tubes each carrying half a unit of D1 charge, and so there is a total of 1 unit of D1 charge escaping. Our entire configuration is then a D1-D5 baryon. While each D3 carried half-integral D1 charge, the D1-charge escaping needed to be integral to satisfy the Dirac quantization condition. The fact that it indeed is integral corresponds to the existence of the division by two in the definition of $P^{1}$.

As in Refs. $[26,33]$ a $\mathrm{D} p-\mathrm{D}(p-4)$ baryon implies that $d_{5}$ of the dual of the $\mathrm{D} p$ brane is the dual of its intersection with the $\mathrm{D}(p-4)$ brane. In our case this reads

$$
d_{5}\left(\alpha \theta_{\alpha}+\beta \theta_{\beta}\right)=\alpha \beta^{2} \theta_{\alpha} \theta_{\beta}
$$




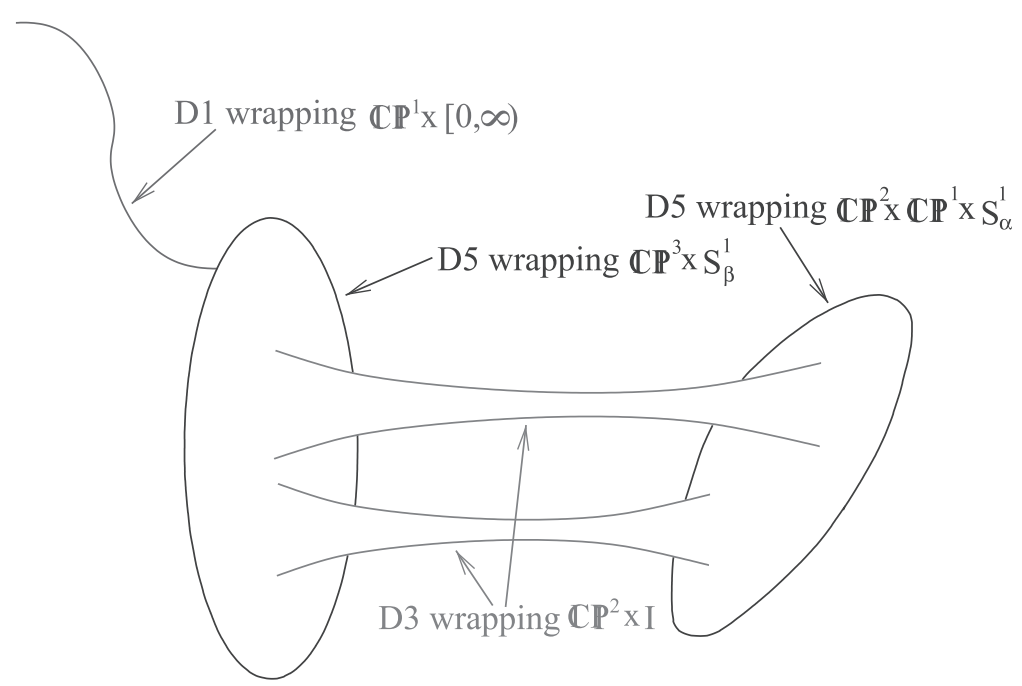

Figure 3: Two D5 components have worldvolume $H$-fluxes that cancel, meaning that one absorbs the D3's sourced by the other. Thus the combination is $d_{3}$ closed, but it is not $d_{5}$ closed. This means that the pair of D5's requires a $\mathrm{D} 1$ insertion, whose cross-sections are dual to $d_{5}$ of the cocycle dual to the $\mathrm{D} 5$ pair. $\mathrm{D}(p+4)-\mathrm{D} p$ baryons are always dual to cycles related by $d_{5}$.

Notice that this agrees with the formula for $d_{5}$ conjectured in Ref. [26]

$$
\begin{aligned}
d_{5}\left(\alpha \theta_{\alpha}+\beta \theta_{\beta}\right) & =\left(\mathrm{sq}^{2} \frac{H}{2}\right)\left(\alpha \theta_{\alpha}+\beta \theta_{\beta}\right)=\left(\mathrm{sq}^{2}\left(\alpha \theta_{\alpha}+\beta \theta_{\beta}\right)\right)\left(\alpha \theta_{\alpha}+\beta \theta_{\beta}\right) \\
& =\beta^{2} \theta_{\beta}\left(\alpha \theta_{\alpha}+\beta \theta_{\beta}\right)=\alpha \beta^{2} \theta_{\alpha} \theta_{\beta} .
\end{aligned}
$$

As always we may interpret the two factors of the differential as the two steps connecting the D1 and the D5. First the $H$ takes the D5-brane to the two D3 tubes, then the $\mathrm{sq}^{2} / 2$ calculates the D1 charge of each tube. This is the same factor of 2 that came into the two step brane within brane embedding in the solution of the extension problem for $\mathbb{R} P^{7}$. Perhaps when there are $n$ steps of embedding there is always a factor of $(n !)$ that counts the orderings of the embeddings.

This story proceeds analogously if we multiply our class by a factor of $\beta$, so that each brane that wrapped $\mathbb{C} P^{j} \subset \mathbb{C} P^{3}$ now wraps $\mathbb{C} P^{j-1} \subset \mathbb{C} P^{3}$. We then find

$$
\begin{aligned}
d_{5}\left(\alpha \beta \theta_{\alpha}+\beta^{2} \theta_{\beta}\right) & =\left(\operatorname{sq}^{2} \frac{H}{2}\right)\left(\alpha \beta \theta_{\alpha}+\beta^{2} \theta_{\beta}\right) \\
& =\beta^{2} \theta_{\beta}\left(\alpha \beta \theta_{\alpha}+\beta^{2} \theta_{\beta}\right)=\alpha \beta^{3} \theta_{\alpha} \theta_{\beta},
\end{aligned}
$$

which again is in agreement with Ref. [26]. 
Restricting to the kernel of $d_{5}$ now kills two of the seven factors of $\mathbb{Z}_{2}$, and another two factors are killed when we quotient by the image. In all we lose two $\mathbb{Z}_{2}$ 's from each associated graded $K$-group and thus the quotient of the kernel of $d_{5}$ by its image is the same associated graded $K$-group found for its $T$-dual in equation (3.15). The extension problems proceed identically, and so the twisted $K$-theory of $\widehat{M}$ agrees with the untwisted $K$-theory of its $T$-dual $\mathbb{R} P^{3} \times \mathbb{R} P^{7}$. Note that here the secondary operation, $d_{5}$, is $T$-dual to the primary operation $d_{3}$. Secondary operations are notoriously difficult to calculate, but this example suggests the possibility that in some classes they may be calculable as primary operations on an auxiliary space. For example one may try to find a recursive relation satisfied by Massey products.

\subsection{The twisted case: $S^{3} \times S^{2 k+1} \times S^{6-2 k}$}

$\mathbb{R} P^{2 k+1}$ is a circle bundle over $\mathbb{C} P^{k}$ with Chern class equal to two. Thus $\mathbb{R} P^{3} \times \mathbb{R} P^{2 k+1} \times S^{6-2 k}$ is a 2 -torus bundle over $\mathbb{C} P^{1} \times \mathbb{C} P^{k} \times S^{6-2 k}$. We claim that T-dualizing two particular generators of this torus, in the presence of the above torsion $H$-flux (equation (3.23)), yields IIB string theory on $S^{3} \times S^{2 k+1} \times S^{6-2 k}$ with no $H$-flux. We will ignore the spheres $S^{6-2 k}$ which support neither the $H$-flux nor the curvature of the circle bundles.

We begin by $T$-dualizing the circle $S_{a}^{1}$ fiber in $\mathbb{R} P^{3}$

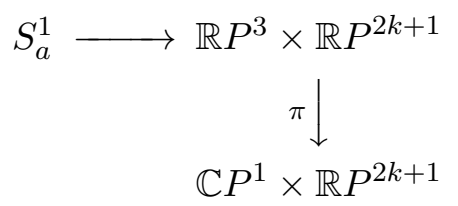

which has Chern class $2 \alpha$, where $\alpha$ is the generator of $H^{2}\left(\mathbb{C} P^{1}\right)=\mathbb{Z}$. The circle $S_{a}^{1}$ is trivially fibered over the $\mathbb{R} P^{2 k+1}$, but the $\mathbb{R} P^{2 k+1}$ is included in this diagram because the $T$-dual circle $\widehat{S}_{a}^{1}$ will be nontrivially fibered over it. As always the $T$-dual fibration is defined by setting the first Chern class equal to the pushforward of the original $H$-flux

$$
\widehat{F}=\pi_{!} H=\pi_{!}\left(a b^{2}+a^{2} b\right)=\int_{S_{a}^{1}} H
$$

The pushforward is just the integral of a differential form representative of $H$ (which by an abuse of notation we have also called $H$ above) over the fiber $S_{a}^{1}$. Although this three-form is exact, it is the derivative of a two-class that does not satisfy the quantization condition (it is half-integral) and so this integral will give a two-form on $S^{2} \times \mathbb{R} P^{2 k+1}$ which is again the derivative of a form that does not satisfy the quantization condition. There are many differential form representatives of $H$ that differ by exact forms that are derivatives of forms that do satisfy the quantization condition, for example 
three times the representative chosen. However we will see that the answer is a $\mathbb{Z}_{2}$ cohomology class and so is unaffected by the addition of even classes.

While we could construct an explicit representative and do the integral, an easier approach is to evaluate the pushforward by equating it with the homology-cohomology pairing with $\mathbb{Z}_{2}$ coefficients. ${ }^{7}$ Then the pushforward is just the pairing with the generator $\langle a|$ of $H_{1}\left(\mathbb{R} P^{3} ; \mathbb{Z}_{2}\right)$, which is our fiber. We then lift our result back to the integral cohomology of the base $\mathbb{C} P^{1} \times$ $\mathbb{R} P^{2 k+1} \times S^{6-2 k}$. Thus

$$
\widehat{F}=\pi !\left(a b^{2}+a^{2} b\right)=\left\langle a \mid a b^{2}+a^{2} b\right\rangle=\left\langle a \mid a b^{2}\right\rangle+\left\langle a \mid a^{2} b\right\rangle=b^{2}+2 a b=b^{2},
$$

where $2 a b$ vanishes because $a$ is $\mathbb{Z}_{2}$-valued. The vanishing of this term was necessary because $a b$ does not lift to an integral class in $H^{2}\left(\mathbb{R} P^{3} \times \mathbb{R} P^{2 k+1}\right)$. $b^{2}$ is the nontrivial class in $H^{2}\left(\mathbb{R} P^{2 k+1}\right)=\mathbb{Z}_{2}$ and so our dual circle $\widehat{S}_{a}^{1}$ is nontrivially fibered over $\mathbb{R} P^{2 k+1}$ and is trivially fibered over the $\mathbb{C} P^{1} \times$ $S^{6-2 k}$

$$
\begin{array}{r}
\widehat{S}_{a}^{1} \longrightarrow \mathbb{C} P^{1} \times S^{2 k+1} \times \widehat{S}_{d}^{1} \times S^{6-2 k} \\
\hat{\pi} \downarrow \\
\mathbb{C} P^{1} \times \mathbb{R} P^{2 k+1} \times S^{6-2 k} .
\end{array}
$$

Here we have used the fact that the unique nontrivial $\widehat{S}_{a}^{1}$ bundle over $\mathbb{R} P^{2 k+1}$ is topologically just $S^{2 k+1} \times \widehat{S}_{d}^{1}$.

The relationship between the two circles $\widehat{S}_{a}^{1}$ and $\widehat{S}_{d}^{1}$ will be crucial later and so we will describe it. The $\widehat{S}_{d}^{1}$ action may be seen in the $\widehat{S}_{a}^{1}$ bundle picture as the diagonal action of $\widehat{S}_{a}^{1}$ and the free circle action whose orbits are each $\mathbb{R} P^{1} \in \mathbb{R} P^{2 k+1}$. This diagonal action is free and has a space of orbits $S^{2 k+1}$ instead of the original $\mathbb{R} P^{2 k+1}$. Each orbit of $\widehat{S}_{a}^{1}$ is twice as long as an orbit of $\widehat{S}_{d}^{1}$, because after circumnavigating one $\widehat{S}_{d}^{1}$ orbit (which would take it back to where it started) the $\widehat{S}_{a}^{1} \propto \widehat{S}_{d}^{1}+\mathbb{R} P^{1}$ orbit has also traveled around the lift of the noncontractible loop $\mathbb{R} P^{1}$, and so it is on the opposite side of sphere. ${ }^{8}$ Thus the $\widehat{S}_{a}^{1}$ orbit only closes after the second loop. An $\widehat{S}_{a}^{1}$ orbit then consists of two $\widehat{S}_{d}^{1}$ orbits plus two trips around $\mathbb{R} P^{1}$. The $\mathbb{R} P^{1}$ generates $H_{1}\left(\mathbb{R} P^{2 k+1}\right)=\mathbb{Z}_{2}$ and so two trips around the $\mathbb{R} P^{1}$ may be deformed into none. We could have predicted in advance that, except for the factor of 2 , our two loops would be homotopic because the fundamental group of $S^{2 k+1} \times S^{1}$ is one-dimensional (figure 4 ).

\footnotetext{
${ }^{7}$ Alternatively the pushforward of $H$ may be calculated by using the exactness of the Gysin sequence for this circle bundle.

${ }^{8}$ This is the reason that antipodal points on the sphere are identified in the space of $\widehat{S}_{a}^{1}$ orbits.
} 


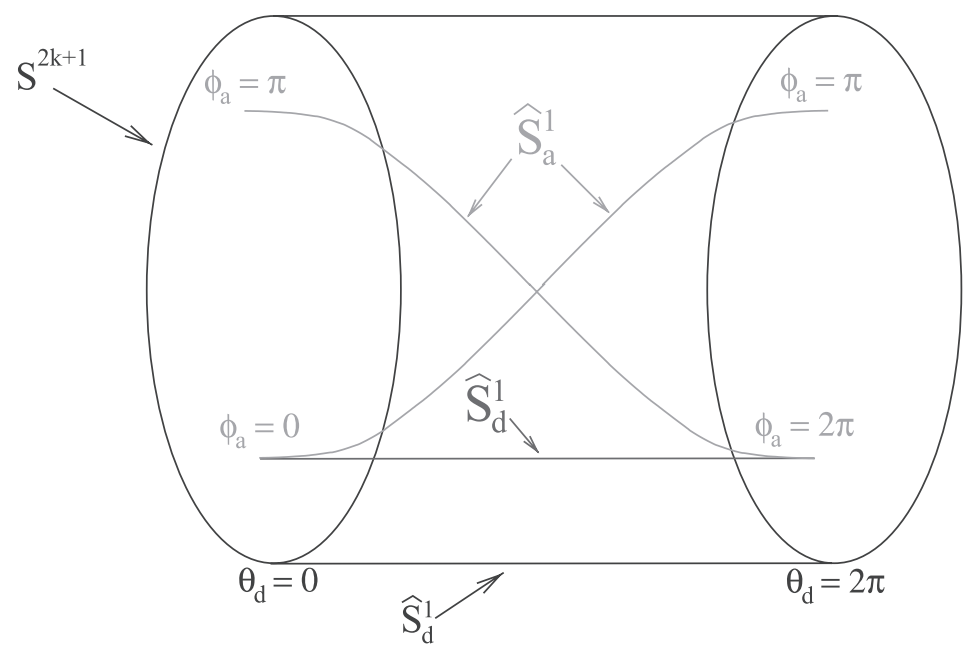

Figure 4: The space $S^{2 k+1} \times \widehat{S}_{d}^{1}$ admits two different free circle actions. One circle action simply goes around the $\widehat{S}_{d}^{1}$ and so has a space of orbits $S^{2 k+1}$. The other has the same action plus the free circle action on the $S^{2 k+1}$, so by the time it has gone around $\widehat{S}_{d}^{1}$ once it is on the other side of $S^{2 k+1}$. This orbit, called $\widehat{S}_{a}^{1}$, therefore does not close until it has gone around $\widehat{S}_{d}^{1}$ twice, when it has returned to its starting point on the sphere. At a given point on $\widehat{S}_{d}^{1}$ the orbit $\widehat{S}_{a}^{1}$ intersects two antipodal points on the $S^{2 k+1}$, and so the space of $\widehat{S}_{a}^{1}$ orbits is the space of pairs of antipodal points, $\mathbb{R} P^{2 k+1}$.

The dual $H$-flux, $\widehat{H}$, is determined from the curvature of the original $S_{a}^{1}$ bundle, which had a Chern class of 2 times the generator $\alpha$ of $H^{2}\left(\mathbb{C} P^{1}\right)=\mathbb{Z}$, via the condition [23]

$$
2 \alpha=F=\hat{\pi}_{!} \widehat{H}=\int_{\widehat{S}_{a}^{1}} \widehat{H}
$$

and the fact that the parts of $H$ and $\widehat{H}$ in the cohomology of the base $\mathbb{C} P^{1} \times \mathbb{R} P^{2 k+1}$ must agree. The original $H$ did not contain any terms in the cohomology of the base, and so neither will the dual $\widehat{H}$. Thus $\widehat{H}$ is simply

$$
\widehat{H}=2 \alpha \cup \theta_{a},
$$

where $\theta_{a}$ generates $H^{1}\left(\widehat{S}_{a}^{1}\right)=\mathbb{Z}$.

This is the answer, but to use the formalism of Ref. [23] to perform the next $T$-duality we will rewrite $\widehat{H}$ in terms of $\widehat{S}_{d}^{1}$, whose first cohomology generator will be named $\theta_{d}$. As $\widehat{S}_{a}^{1}$ is homotopic to twice $\widehat{S}_{d}^{1}$ the cohomology generator $\theta_{d}$ must be cohomologous to twice the generator $\theta_{a}$. This factor 
of two is necessary to preserve the homology-cohomology pairing, or equivalently the fact that the cohomology classes need to integrate to one over their corresponding cycles.

Note that while as a cohomology class

$$
\left[\theta_{d}\right] \sim 2\left[\theta_{a}\right]
$$

it is in fact crucial, even to the topology of the space of orbits of the circle action, that they differ by the single unit free circle action on the $S^{2 k+1}$, or equivalently by two units of the generator of $H_{1}\left(\mathbb{R} P^{7}\right)$.

Summarizing, the first $T$-duality has left us with $\mathbb{C} P^{1} \times S^{2 k+1} \times \widehat{S}_{d}^{1} \times$ $S^{6-2 k}$ with an NS-flux

$$
\widehat{H}=2 \alpha \cup \theta_{a}=\alpha \cup \theta_{d}+\alpha d \phi \sim \alpha \cup \theta_{d},
$$

where $d \phi$ is the cohomologically trivial unit generator of the free circle action on the $S^{2 k+1}$. The last equality is an equality of the cohomology classes that follows from the cohomological triviality of $d \phi$, but we will return to the $d \phi$ term later. Note that while shifting $H$ by an exact form $d B$ does not change its topology, it does change the gauge-invariant Wilson loops $\int B$ and so may have an effect on the physics. We have in effect changed the background that we are considering. We will claim that in the case $k=3$ a consistent and a potentially inconsistent compactification are related by such an exact shift.

The second $T$-duality is much easier. We now want to $T$-dualize the circle $\widehat{S}_{d}^{1}$, and so we will have $T$-dualized all of our original $T^{2}$ bundle over $\mathbb{C} P^{1} \times \mathbb{C} P^{k}$, but in a nonorthogonal basis. If we $T$-dualized about $\widehat{S}_{a}^{1}$ we would return to where we started. Despite the fact that $\widehat{S}_{a}^{1}$ is homotopic to a multiple of $\widehat{S}_{d}^{1}$ on the total space, it yields a topologically inequivalent circle bundle ${ }^{9}$ and we will see that it leads to a topologically inequivalent $T$-dual. However, as the $T$-duals with respect to $\widehat{S}_{a}^{1}$ and $\widehat{S}_{d}^{1}$ are related to each other by two $T$-dualities they will necessarily have isomorphic $K$-theories twisted with respect to their corresponding $H$-fluxes.

The circle $\widehat{S}_{d}^{1}$ is trivially fibered over the base $\mathbb{C} P^{1} \times S^{2 k+1} \times S^{6-2 k}$ with an $H$-flux that contains no component entirely on the base, thus the dual $H$-flux is trivial. The fibration of the dual $\widehat{\widehat{S}^{1}}$ over $\mathbb{C} P^{1} \times S^{2 k+1} \times S^{6-2 k}$ is

\footnotetext{
${ }^{9}$ For example the spaces of orbits, $S^{2 k+1}$ and $\mathbb{R} P^{2 k+1}$, are not homeomorphic.
} 
described by the Chern class

$$
\widehat{\widehat{F}}=\int_{\widehat{S}_{d}^{1}} \widehat{H}=\int_{\widehat{S}_{d}^{1}} \alpha \cup \theta_{d}=\alpha
$$

which is the generator of $H^{2}\left(\mathbb{C} P^{1}\right)=\mathbb{Z}$. This identifies the dual bundle as the Hopf fibration over our 2 -sphere, whose total space is the 3 -sphere. The curvature $\widehat{\widehat{F}}$ contains no component on the $(2 k+1)$-sphere, and so our resulting spacetime is topologically a Cartesian product of spheres

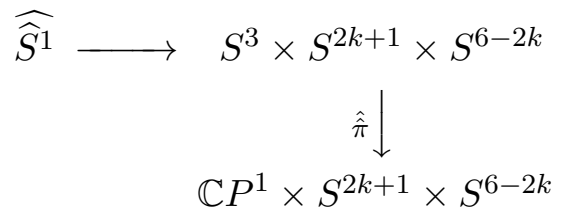

with no $H$-flux. As there is no $H$-flux the resulting twisted $K$-theory is just the untwisted $K$-theory of $S^{3} \times S^{2 k+1} \times S^{6-2 k}$ which is isomorphic to its integral cohomology, as there is no torsion

$$
K^{0}\left(S^{3} \times S^{2 k+1} \times S^{6-2 k}\right)=K^{1}\left(S^{3} \times S^{2 k+1} \times S^{6-2 k}\right)=\mathbb{Z}^{2} \oplus \mathbb{Z}^{2}=\mathbb{Z}^{4} .
$$

Note the crucial role played by the factor of two difference in the two circles $\widehat{S}_{a}^{1}$ and $\widehat{S}_{d}^{1}$. Had the corresponding 1-cycles not had a relative factor of two difference in their normalization then there would have been two units of $\widehat{H}$ and so two units of $\widehat{F}$ yielding $\mathbb{R} P^{3} \times S^{2 k+1} \times S^{6-2 k}$ which would have

$$
K^{0}\left(\mathbb{R} P^{3} \times S^{2 k+1} \times S^{6-2 k}\right)=\mathbb{Z}^{4} \oplus \mathbb{Z}_{2}^{2}
$$

We would not have been able to eliminate this possibility using the AHSS computation of the original twisted $K$-theory as it would correspond to the other solution of the extension problem (3.30). Thus the first map $f$ of the extension seems to be multiplication by two because, like in the case of the computation of the twisted $K$-theory of bundles over a single $\mathbb{R P}^{n}$, the $T$ dual orbit $\left(\widehat{S}_{a}^{1}\right)$ is twice as long as the shortest orbit $\left(\widehat{S}_{d}^{1}\right)$. This new shortest element is not in the image of $f$, which only arrives at multiples of $\widehat{S}_{a}^{1}$. Thus we may think of the short element $\widehat{S}_{d}^{1}$ as the nontrivial element in the image of $g, 1 \in \mathbb{Z}_{2}$. The extension would have split, yielding the other result for the twisted $K$-theory of $\mathbb{R} P^{3} \times \mathbb{R} P^{2 k+1} \times S^{6-2 k}$ if instead there had been no relation between the loops $\widehat{S}_{a}^{1}$ and $\widehat{S}_{d}^{1}$ and they had formed separate classes in $K^{1}$, which is $T$-dual to $K^{0}\left(\mathbb{R} P^{3} \times \mathbb{R} P^{2 k+1} \times S^{6-2 k}\right)$. 
5 D3-brane insertion on $\mathbb{R} P^{1} \times \mathbb{R} P^{2 k-3} \times S^{6-2 k}$

\subsection{The $S$-dual configuration with torsion $G_{3}$}

While the twisted $K$-theories of all of the spaces above are well-defined, $\mathbb{R} P^{3} \times \mathbb{R} P^{2 k+1} \times S^{6-2 k}$ configurations with torsion $H$-flux

$$
H=a b^{2}+b a^{2},
$$

do not correspond to $S$-covariant $K$-theory classes when $k>1$ because

$$
S q^{3} H=H \cup H=a^{2} b^{4} \neq 0 .
$$

For example if the other fluxes $G_{2 j+1}$ all vanish then, if we implement $S$-duality by simply interchanging the integral classes $G_{3}$ and $H$, we find that now the $S$-dual fields are

$$
\tilde{H}=0, \quad \tilde{G}_{3}=a b^{2}+a^{2} b,
$$

and so there is a Freed-Witten anomaly

$$
d_{3} \tilde{G}_{3}=\left(S q^{3}+\tilde{H} \cup\right) \tilde{G}_{3}=S q^{3} \tilde{G}_{3}=a^{2} b^{4} .
$$

This is to be expected, compact flux configurations in the presence of branes do not correspond to $K$-theory classes because the fluxes are not closed under the AHSS differentials. In this case the unit of D3 charge

$$
Q_{\mathrm{D} 3}=d_{3} \tilde{G}_{3}=a^{2} b^{4}=1 \in H^{6}\left(\mathbb{R} P^{3} \times \mathbb{R} P^{2 k+1} \times S^{6-2 k}\right)=\mathbb{Z}_{2}^{2},
$$

is dual to the nontrivial element of the $\mathbb{Z}_{2}$-valued homology cycle

$$
H_{4}\left(\mathbb{R} P^{1} \times \mathbb{R} P^{2 k-3} \times S^{6-2 k}\right)
$$

In short, this $S$-dual story is a typical application of the Freed-Witten anomaly. In addition there may be additional D-branes required to cancel tadpoles of a purely gravitational origin, such as the D3-brane dual to $P$.

However if $k=3$ then

$$
\tilde{G}_{3} \cup \tilde{G}_{3} \cup \tilde{G}_{3}=a^{3} b^{6} \neq 0,
$$

and so the D3-brane, which is PD to $\tilde{G}_{3} \cup \tilde{G}_{3}$, itself supports $\tilde{G}_{3}$-flux equal to the torsion generator $a b^{2}$ of $H^{3}\left(\mathbb{R} P^{1} \times \mathbb{R} P^{3}\right)=\mathbb{Z} \oplus \mathbb{Z}_{2}$, where we have dropped the unimportant $S^{0}$ factor. This is $S$-dual to a D3-brane that supports torsion $H$-flux, which leads to a Freed-Witten anomaly that forces the D3-brane to source a single D-string that wraps the dual $\mathbb{R} P^{1} \subset \mathbb{R} P^{3}$. In this case the D3-brane worldvolume action term

$$
S_{D 3} \supset \int C_{2} \wedge * F
$$

implies that a D3-brane supporting a unit of $\tilde{G}_{3}$-flux has a unit of electric charge on its worldvolume gauge theory. 
The D3-brane is compact and so the total charge must be canceled, which requires the insertion of a fundamental string, whose endpoint on a D-brane is an electric charge. The fundamental string, like its $S$-dual, wraps $\mathbb{R} P^{1}$ and extends away from the D3-brane. However our total spacetime is compact and so this $F$-string must have two ends. Yet there is no obvious candidate for another brane on which this string may end without creating another anomaly, since there are no nontrivial $K$-classes corresponding to the potential wrappings of the required 1 or 5 -brane baryons. Thus the configuration is apparently inconsistent once we take into account the $S$-dual Freed-Witten anomalies, although we cannot rule out the possibility that some more complicated anomaly allows this $F$-string to be absorbed. For example if $P$ contains a term $a^{2} b^{4}$ then there will be a gravitational D3 wrapping the same cycle as the D3-brane above, which will cancel its D3brane charge or equivalently supply a second endpoint for the string and so restore the consistency of the configuration.

\subsection{Torsion $H$}

In the story of interest, however, it is not $G_{3}$ whose square is nontrivial but rather $H$. This means that we need to replace equation (5.4) with the $S$-dual Freed-Witten anomaly. This is the Freed-Witten anomaly as derived for the worldvolume of an NS5-brane instead of a D5-brane. The usual derivation of the anomaly relies upon the use of the worldsheet of a perturbative string which can not obviously be made Bogomol'nyi-PrasadSommerfield (BPS). An application of $S$-duality to that argument may force us to go to a region of moduli space where the strong coupling effects render such perturbative strings unavailable, although in the large volume limit this calculation should nonetheless be reliable [20]. And so rather than trying to $S$-dualize the argument of Freed and Witten, we will instead define the action of $S$-duality on torsion elements of integral cohomology by extending its action upon the elements of real cohomology $d B$ and $d C_{k}$. That is, we will say that $S$-duality exchanges the integral classes $H$ and $G_{3}$. We will then conjecture that IIB string theory is $S$-duality covariant and search for inconsistencies.

When $k>1$ our configuration with torsion $H \neq 0$ and $G=0$ corresponds to a twisted $K$-theory class in $K_{H}^{1}$ but not to a class in the untwisted $K$-theory $K_{\tilde{H}=G_{3}=0}^{1}$ with torsion $\tilde{G}_{3}=H$ due to the anomaly (5.4). In particular our configuration has a Freed-Witten anomaly which is $S$-dual to equation (5.4)

$$
Q_{\mathrm{D} 3}=d G_{5}=d_{3} H=H \cup H=a^{2} b^{4},
$$


leading again to a D3-brane supported on $\mathbb{R} P^{1} \times \mathbb{R} P^{2 k-3} \times S^{6-2 k}$. Again we are not considering the possible gravitational correction $P$ to $Q_{\mathrm{D} 3}$. D3-branes are invariant under $S$-duality, and according to our conjecture $\mathbb{Z}_{2}$-charged D3-branes are invariant too. Thus we have found that the brane that cures this anomaly is just the $S$-dual of the brane that cured the $S$-dual anomaly above, which is not surprising as our formula for D3 charge is $S$-duality invariant.

As another check we can see what happens to the system when the $H$-flux is turned on or off. An $H$-flux can be turned on or off by passing an NS5brane that sources it. For example in Ref. $[33,36]$ where twisted $K$-theory classified fluxes at moments in time, an NS5-brane that sweeps out a 6-cycle during some interval of time will change the $H$-flux on the dual cycle. If the cycle contains $G_{3}$ or if its normal bundle is not $\operatorname{spin}^{c}$ then the NS5brane worldvolume will have an anomaly that is canceled by a D3-brane insertion, making a baryon [10] configuration in which an NS5-brane sweeps out a cycle and a D3-brane ends on the NS5-brane and continues toward an infinity in the time direction. Thus the initial and final conditions differ by a unit of $H$-flux and also possibly some D3-brane charge which is equal to the anomaly on the NS5-brane worldvolume and is approximated by equation (5.9). In fact such instantons interpolate between brane configurations that are homologically distinct but represent the same twisted $K$-homology classes in the $S$-dual twisted $K$-homology $K_{G_{3}}$.

In our case there is no time direction. We have used all 10 directions to construct $\mathbb{R} P^{3} \times \mathbb{R} P^{2 k+1} \times S^{6-2 k}$. However we are not using $K$-theory to find the conserved charges on a timeslice. Rather we are using $K$-theory to classify configurations on all of spacetime, as in Ref. [2]. In that paper, rather than transforming between different cohomological representatives of the same $K$-class on different timeslices via instantons that act over an interval of time, the author uses Sen's construction [37] to transform between cohomological representatives of the same $K$-class on the whole of spacetime via instantons that act over an interval in a deformation direction that is not one of the 10 dimensions of the spacetime. The deformation direction in this case corresponds to the RG flow of the string field theory undergoing tachyon condensation. Similarly the relation between different representatives of the same class of the $S$-dual twisted $K$-theory of the deformed conifold was seen to be an RG flow, the Klebanov-Strassler cascade, in Ref. [16]. In short, the RG flow means that we can introduce an extra non-physical deformation dimension in which we may place Maldacena-Moore-Seiberg (MMS) instantons that transform between distinct cohomology representatives of the same twisted $K$-theory class. The $K$-classes may then be identified with the universality classes of the theory whose flow we have used. The deformation direction appeared similarly in Ref. [32]. 
Now we may toggle the $H$-flux by wrapping an NS5-brane around the oriented $\mathbb{Z}_{2}$-valued cycle of $H_{7}\left(\mathbb{R} P^{3} \times \mathbb{R} P^{2 k+1} \times S^{6-2 k}\right)$ that came from the Tor term in the Künneth formula. This cycle is the PD of $a b^{2}+b a^{2}$, which is just the dual of $a b^{2}, \mathbb{R} P^{2} \times \mathbb{R} P^{2 k-1} \times S^{6-2 k}$, glued to the dual of $a^{2} b, \mathbb{R} P^{1} \times$ $\mathbb{R} P^{2 k} \times S^{6-2 k}$, along their common $\mathbb{R} P^{1} \times \mathbb{R} P^{2 k-1} \times S^{6-2 k}$, which is cut in each component to make the components orientable. Note that the NS5brane spans 7 dimensions, instead of the usual 6 , in fact all branes extend in one extra dimension now that we have included the deformation direction. However the NS5-brane does not extend in the deformation direction, which is the reason that as we pass the NS5-brane in the deformation direction the $H$-flux changes, toggling the $\mathbb{Z}_{2} \subset H^{3}\left(\mathbb{R} P^{3} \times \mathbb{R} P^{2 k+1} \times S^{6-2 k}\right)$ on or off.

When $k>1 H \cup H \neq 0$ implies that this NS5-brane is anomalous. This is the reason that the flux that it sources suffers from a Freed-Witten anomaly. The NS5-brane wraps a cycle whose normal bundle is not $\operatorname{spin}^{c}$, and so it may only be rendered consistent if it also sources a $\mathbb{Z}_{2}$ charged D3-brane. This 5-dimensional D3-brane ends with codimension 3 on the NS5-brane and continues to plus or minus infinity in the deformation direction. Thus the D3-brane charge, which itself is $\mathbb{Z}_{2}$ valued, is toggled at the same time as the $H$-flux is toggled. When $H$ vanishes the D3 charge needs to vanish (more generally it is equal to $P$ ) because D3 is a source for $d C_{4}$ which is gauge invariant in the absence of $H$-flux and so the integral of $d d C_{4}$, the total D3-brane charge, must vanish over the boundaryless $\mathbb{R} P^{3} \times \mathbb{R} P^{2 k-3} \times S^{6-2 k}$ by Stokes' theorem. If the D3-charge is zero when $H$ is zero and the D3charge changes when $H$ changes then the D3-charge must be $1 \in \mathbb{Z}_{2} \subset H_{4}$ when $H$ is $1 \in \mathbb{Z}_{2} \subset H^{3}$. In conclusion we have again used the $S$-dual FreedWitten anomaly, this time on the worldvolume of the NS5-brane, and again we have found the same result, that our configuration with $H$ flux has a unit of D3-brane charge wrapped on $\mathbb{R} P^{1} \times \mathbb{R} P^{2 k-3} \times S^{6-2 k}$.

\section{T-dualizing to D2}

\subsection{The T-duality}

We have found that $\mathbb{Z}_{2}$-valued D3-branes, one of which may be inconsistent, are required for anomaly cancellation in the cases $k=2$ and $k=3$. These branes cannot be removed without also removing the $H$-flux. However the $T$-duals, in particular the fluxless product of spheres, have no Freed-Witten anomalies and so the above restriction appears to have disappeared. This means that during the course of $T$-dualizing the D3-branes need to disappear. In this section we provide a proposal for how the brane charge might 
change. Notice that if $P=a^{2} b^{4}$ then the branes are simply canceled by the gravitational branes before any dualities.

In the case $k=3$ the D3-brane is a baryon which sources a D-string as it wraps nontrivial $H$-flux. Its $T$-dual is therefore a kind of baryon as well, as the D-string dualizes to a D2-brane that ends on our dual D2. The twisted $K$-theory classification does not include D-brane-D-brane baryons [36] and so this brane does not dualize to a class in twisted $K$-theory. We do not need to use the claim that the compactification is inconsistent in this argument. However the fact that the baryon has a brane of the same dimension ending it suggests the seriousness of the topological obstruction confronting it. In fact it wraps a hemisphere that has a boundary, and so the D2-brane ending on it is the brane continuing out from that boundary.

The case $k=2$ is more interesting. Although $\mathbb{R} P^{5}$ is not spin, the $T$ duality to $S^{3} \times S^{5} \times S^{2}$ without fluxes ensures that the spectrum of the full string theory is supersymmetric, as in Ref. [21]. As noted in the introduction, since the dual spheres are small the $T$-duality does not prove that string theory can be defined on this non-spin space. ${ }^{10}$ If it cannot then we need to consider the $\mathbb{R} P^{5}$ to be an orientifold. This would change the classification of fluxes. For example, if we replace the $S^{2}$ by a $T^{2}$ then the desired orientifolding may be achieved with an O3-plane that wraps the $\mathbb{R} P^{3}$ and a circle in the torus. In this case the three and seven-dimensional field strengths will be classified by $\mathbb{Z}_{2}$-twisted cohomology $[10,22]$ and in particular will be $\mathbb{Z}_{2}$-valued. The five class, under which our D3 is charged, will still be classified by integral cohomology. However it is no longer clear that our $H$-flux is coclosed. We will not consider this case further.

While we cannot prove that the twisted $k=2$ string theory exists, we can still study the charges and duality transformations that the D3-brane will have if it indeed does exist. The D3-brane wraps $\mathbb{R} P^{1} \times \mathbb{R} P^{1} \times S^{2} \subset$ $\mathbb{R} P^{3} \times \mathbb{R} P^{5} \times S^{2}$. Recall that we constructed $H=a b^{2}+a^{2} b$, by taking the Bockstein of a two-class $a b$. Thus we identify the 2-class $a b$ with the B-field and use the homology-cohomology pairing to write

$$
\int_{\mathbb{R} P^{1} \times \mathbb{R} P^{1}} B \sim 1
$$

The integral of the $B$-field over a 2-cycle of a $\mathrm{D} p$-brane measures the worldvolume $\mathrm{D}(p-2)$-charge, and so already we see that this D3-brane carries D1-brane charge. This is a consequence of the extension problem, in which

\footnotetext{
${ }^{10}$ The same applies the example of Ref. [21] and so we are optimistic that, using the $H$ flux, it can be defined.
} 
the corresponding

$$
\mathbb{Z}_{2}=H^{2}\left(\mathbb{R} P^{3}\right) \otimes H^{4}\left(\mathbb{R} P^{5}\right) \subset H^{6}\left(\mathbb{R} P^{3} \times \mathbb{R} P^{5}\right),
$$

was absorbed by

$$
\mathbb{Z}=H^{3}\left(\mathbb{R} P^{3}\right) \otimes H^{5}\left(\mathbb{R} P^{5}\right)=H^{8}\left(\mathbb{R} P^{3} \times \mathbb{R} P^{5}\right),
$$

via the extension

$$
H^{8}\left(\mathbb{R} P^{3} \times \mathbb{R} P^{5}\right)=\mathbb{Z} \longrightarrow \mathbb{Z} \longrightarrow \mathbb{Z}_{2} \subset H^{6}\left(\mathbb{R} P^{3} \times \mathbb{R} P^{5}\right) .
$$

In terms of cohomology classes the extension problem set

$$
2 a^{2} b^{4}=a^{3} b^{5}
$$

which, after Poincaré dualizing, means that our D3-brane carries half a unit of charge of D1-brane wrapped around the $S^{2}$. Thus we see that while our D3-brane appears to be torsion, even multiples of the D3 do not decay into nothing, but rather they leave D-strings that wrap the 2-sphere. This sets the normalization of the $B$-flux in equation (6.1) to one half.

If $P=a^{2} b^{4}$ then there will be a second D3 wrapping the same cycle, and so the two D3's will annihilate. The nature of the gravitational anomaly that the D3 cancels will determine its worldvolume D1 charge, which will be halfintegral. Depending on the D1 charges carried by the D3-branes, which are in principle determined entirely by the anomalies of the configuration, there will be some integral number of D1-branes remaining after the annihilation.

Although the $T$-dual has no torsion homology, we may still see the extension problem in action in the dual picture. We have seen (6.1) that the D3-brane wraps a torus $\mathbb{R} P_{a}^{1} \times \mathbb{R} P_{b}^{1}$ that supports a half unit of NS $B$-flux, while its worldvolume $U(1)$ gauge field strength $F$ is quantized. $B+F$ is gauge-invariant and we may formally construct a pseudo-bundle that has Chern class $B+F$. The connection of this bundle $A$ may be integrated over $\mathbb{R} P_{a}^{1}$ at various values of $\phi_{b} \in \mathbb{R} P_{b}^{1}$ to yield a Wilson loop

$$
f\left(\phi_{b}\right)=\int_{\mathbb{R} P_{a}^{1} \times \phi_{b}} A .
$$

As $B+F$ is half-integral, the Wilson loop is not single valued, but rather it shifts by $\pi$ each time one encircles $\mathbb{R} P_{b}^{1}$

$$
f\left(\phi_{b}+2 \pi\right)=f\left(\phi_{b}\right)+\pi
$$

The D3-brane wraps the $\mathbb{R} P_{a}^{1}$ which is $T$-dualized, and so it is dual to a D2-brane that is localized at a point $\hat{\phi}_{a}$ on the dual circle $\widehat{S}_{a}^{1}$. This point is 
determined by the Wilson loop

$$
\hat{\phi}_{a}\left(\phi_{b}\right)=f\left(\phi_{b}\right)
$$

and so each time one encircles $\mathbb{R} P_{b}^{1}$, the brane goes half way around $\widehat{S}_{a}^{1}$. This is just the construction of $\widehat{S}_{d}^{1}$, which is half of $\widehat{S}_{a}^{1}$ plus $\mathbb{R} P_{b}^{1}$, and so the dual D2-brane wraps $S^{2}$ times the $\widehat{S}_{d}^{1}$, which is the generator

$$
\widehat{S}_{d}^{1}=1 \in H_{1}\left(S^{2} \times S^{5} \times S^{1}\right) .
$$

Meanwhile the dual of the D1-brane that wrapped the $S^{2}$ is a D2-brane that wraps $S^{2} \times \widehat{S}_{a}^{1}$. This is because it did not wrap the original circle $\mathbb{R} P_{a}^{1}$ so it must wrap the dual circle $\widehat{S}_{a}^{1}$. However $\widehat{S}_{a}^{1}$ corresponds to the element

$$
\widehat{S}_{a}^{1}=2 \in H_{1}\left(S^{2} \times S^{5} \times S^{1}\right),
$$

and so, as indicated by the extension problem, the D1-brane (which generated $\mathbb{Z}$ ) dualizes to twice the D3-brane (which generated $\mathbb{Z}_{2}$ ). If $P=a^{2} b^{4}$ then the D2 will wrap $\widehat{S}_{a}^{1}$ an integer number of times, or equivalently it will wrap $\widehat{S}_{d}^{1}$ an even number of times.

\subsection{The D2 disappears when a global $B$-field is included}

It seems a bit strange that the $D 2$-brane disappears when a global $B$-field is included. After $T$-dualizing in the case $k=2$ we have found that the $K$-class corresponding to D1-branes on the $S^{2}$ is odd. No such restriction exists on the product of spheres $S^{2} \times S^{5} \times S^{1} \times S^{2}$ with no fluxes that appears after $T$-dualizing $\widehat{S}_{d}^{1}$, and so as a consistency check we will investigate how this condition might disappear. In the process we find a new variant of the Freed-Witten anomaly.

After the first $T$-duality the $H$-flux is

$$
H=2 \alpha \cup \theta_{a},
$$

where $\theta_{a}=d \phi_{a}$ generates $H^{1}\left(\widehat{S}_{a}^{1}\right)=\mathbb{Z}$. To perform the second $T$-duality, along $\widehat{S}_{d}^{1}$, we claim that we need to ${ }^{11}$ decompose $H$ into a part along and transverse to $\widehat{S}_{d}^{1}$. That is, we wish to write $H$ in terms of $\theta_{d}$ instead of $\theta_{a}$. This is no problem topologically, as $2 \theta_{a}$ is cohomologous to $\theta_{d}$, and

\footnotetext{
${ }^{11}$ Otherwise we might expect to find something in the metric and the $B$-field of the dual product of circles $S^{3} \times S^{5} \times S^{2}$ that produces D1 charge on the $S^{2}$, such as a twisted version of an $S$-dual of the 1-loop effect in Ref. [17]. If no such effect exists then this would suggest that $P=a^{2} b^{4}$.
} 
for the twisted $K$-theory automorphism only the cohomology class of $H$ is important $[23,35]$. In particular we may rewrite $H$ as

$$
H^{\prime}=\alpha \cup \theta_{d}
$$

However geometrically $H$ and $H^{\prime}$ differ by an exact form $B$

$$
\Delta H=H-H^{\prime}=\alpha \cup\left(2 \theta_{a}-\theta_{d}\right)=d\left(\left(2 \phi_{a}-\phi_{d}\right) \alpha\right)=d B .
$$

$\widehat{S}_{a}^{1}$ is twice as long as $\widehat{S}_{d}^{1}$, and so the function $2 \phi_{a}-\phi_{d}$ is a stepfunction which is zero the first time around $\widehat{S}_{d}^{1}$ and then $2 \pi$ the second. While $\Delta H$ is exact and so topologically trivial, we will see that it enters an anomaly multiplied by a gauge-dependent term and the product is topologically nontrivial.

To see this we perform a 9-11 flip (this is the same as $T$-dualizing the $S$-dual description of Section 5.1 in which $\tilde{G}_{3} \neq 0$ and $\tilde{H}=0$ ) so that $\widehat{S}_{a}^{1}$ is the $M$-theory circle. Now our IIA spacetime is $\mathbb{C} P^{1} \times \mathbb{R} P^{5} \times S_{m}^{1} \times S^{2}$ where $S_{m}^{1}$ is the former $M$-theory circle. The new $H$ is roughly

$$
H=2 \alpha \cup \theta_{m}
$$

However the $M$-theory lift of $\Delta H$, which is $\Delta G_{4}$, is not independent of the $M$-theory coordinate $\phi_{a}$. On the contrary since it is the derivative of the step function it is a Dirac delta function at $\phi_{a}=0$ and $\phi_{a}=\pi$, with opposite signs at the two values.

Now there is also $G_{2}$-flux

$$
G_{2}=b^{2}
$$

We recall, from the extension problem for the $K$-theory of $\mathbb{R} P^{2 k+1}$, that $G_{2}$-flux carries half a unit of $G_{4}$-flux. That is to say

$$
2 G_{4}=\mathrm{sq}^{2} G_{2}=G_{2} \cup G_{2}=b^{4} .
$$

Now we use the Freed-Witten anomaly

$$
\mathrm{PD}(\mathrm{D} 2)=d d C_{5}=\Delta H \cup G_{4}=\alpha \cup \theta_{m} \cup b^{4},
$$

to conclude that the shift in $H$ by an exact form yields a half D2-brane wrapping $\mathbb{R} P^{1} \times S^{2}$ at $\phi_{a}=0$ and an anti half $\mathrm{D} 2$ at $\phi_{a}=\pi$, the two points where $H$ is nonzero. As $H_{3}\left(\mathbb{R} P^{1} \times S^{2}\right)=\mathbb{Z}_{2}$ there is no topological difference between a D2 and an anti-D2 wrapped on this cycle but the orientation may mean that they carry opposite F-string charges, which are $\mathbb{Z}$-valued. 
The D2-brane worldvolume coupling

$$
S_{\mathrm{D} 2} \supset \int C_{1} \cup B
$$

combined with

$$
1=G_{2}=d C_{1}
$$

implies that half D2-branes each carry half-integral charge of $F$-string wrapped around $S^{2}$. As always, this $F$-string is an M2-brane and wraps the $M$-theory circle, $\widehat{S}_{a}^{1}$. However the relative signs of these two half-strings depend on a lift of their $\mathbb{Z}_{2}$ classes that we have not determined and perhaps it cannot be determined without finding additional consistency conditions satisfied by the strings, although it seems plausible that the lifts of a brane and antibrane cancel, suggesting $P=a^{2} b^{4}$. Thus the total $F$-string charge changes by an amount that depends on their unknown lift. Now doing a 9-11 flip back we find that each $F$-string becomes a D2-brane that wraps $S^{2} \times \widehat{S}_{d}^{1}$, which as required is the $T$-dual of the D3-brane insertion in IIB. If we can calculate the above lift then we will be able to compute change in D-brane charge, which will in turn allow us to compute $P$.

Thus we have learned that the addition of an exact form to $H$ can potentially, due to an $S$-dual composite Freed-Witten anomaly, toggle the D2brane charge that is produced by our $T$-duality. As this addition of the exact form is apparently necessary between the two $T$-dualities if we wish to use the $T$-duality prescription of $[23,24]$, we find that the charge of our D3 insertion may be canceled before we arrive at $S^{3} \times S^{5} \times S^{2}$ with no flux. We feel that this result, despite our inability to compute the lifts, teaches us about the limitations of the $K$-theory program. The $K$-theory classification relies heavily on forgetting the Wilson loops. But here we see that the Wilson loops may be able to affect the D-brane charges.

It may seem as though we have only replaced one problem with an equivalent problem. After $T$-dualizing from $\mathbb{R} P^{3} \times \mathbb{R} P^{7} \times S^{2}$ we found that the original D-brane insertion implied that the brane charge is, up to gravitational corrections, an odd element of the $\mathbb{Z}=K_{1}\left(\mathbb{C} P^{1} \times S^{5} \times S_{d}^{1} \times S^{2}\right)$. This did not seem to agree with the physics of the final spacetime $S^{3} \times$ $S^{5} \times S^{2}$, but we found that before doing the second $T$-duality we need to include a globally defined $B$-field that can change our $K_{1}$ class by one unit in $\mathbb{Z}$. Now the $K$-homology class is even instead of odd, and so we may ask again how this restriction arises from the viewpoint of the product of spheres. The answer, as was explained in the introduction, is that because our spacetime is compact the only allowed $K$-homology class for D-brane 
wrappings is zero ${ }^{12}$, which is even and so there is no contradiction. To get branes filling out the entire twisted $K$-homology we may, for example, replace the 2 -sphere with a noncompact space. In this case the $K$-class of the product of spheres may be either odd or even. Correspondingly, the original product of projective spaces may now have a $d F$ type D3-brane that wraps $\mathbb{R} P^{1} \times \mathbb{R} P^{1} \subset \mathbb{R} P^{3} \times \mathbb{R} P^{5}$ and cancels the D3 charge but preserves the consistency of the configuration. Thus in the noncompact case there may be any net D3 charge in the initial configuration and so the $K$-class may be odd or even in the $T$-dual configuration, while in the compact case the original D3-charge is 1 corresponding to a final $K$-class which is 0 . In both cases we have then seen that the allowed charges before and after the $T$-duality can agree, as they must.

\subsection{A correction to the $W_{7}$ anomaly}

The anomaly used in this argument may be summarized as

$$
\mathrm{PD}(F-\text { String })=d d B_{6}=H \cup G_{2} \cup G_{2} \cup C_{1} \text {. }
$$

At an intermediate step, to construct the D2-brane, we used a simpler anomaly

$$
\mathrm{PD}(\mathrm{D} 2)=d d C_{5}=H \cup G_{2} \cup G_{2} .
$$

This appears to be an extra term in the DMW anomaly [4]

$$
W_{7}=0 \text {. }
$$

Equation (6.22) is a special case of the Freed-Witten anomaly

$$
\mathrm{PD}(\mathrm{D} 2)=d d C_{5}=\left(S q^{3}+H \cup\right) G_{4},
$$

because when $H$ vanishes and there are no D2-branes the flux quantization condition on spin manifolds

$$
G_{4}=w_{4} \bmod 2
$$

yields the DMW anomaly

$$
W_{7}=S q^{3}\left(w_{4}\right)=S q^{3} G_{4}=0 .
$$

The flux quantization condition (6.24) has been demonstrated on spin manifolds, and the current spacetime is not spin. For non-spin manifolds counterexamples are known $[21,23]$. In this case in fact it holds because

$$
w_{4}\left(\mathbb{R} P^{5}\right)=\left(\begin{array}{l}
6 \\
4
\end{array}\right) \quad \bmod 2=1,
$$

\footnotetext{
${ }^{12}$ More precisely we argued that it is the class for which there are only Hanany-Witten type brane charges. Thus the allowed class is odd before we change the $B$-field, 0 after, and 0 on the final $S^{3} \times S^{5} \times S^{2}$.
} 
and $G_{4}$ is nontrivial as $G_{2}$ produces a half unit of $G_{4}$-flux via $G_{2} \cup G_{2}$. Thus the applicable quantization condition in this non-spin case may include a contribution

$$
G_{2} \cup G_{2} \bmod 2 \text {, }
$$

which (since $S q^{3} G_{2}=0$ ) leads to a contribution to the D2 charge of $H \cup$ $G_{2} \cup G_{2}$. Combining these contributions suggests that the mod 2 part of the D2-charge may need a correction. For example when the $M$-theory manifold is spin then $G_{2}=w_{2} \bmod 2$ and so one may expect an $H \cup w_{2} \cup w_{2}$ contribution.

\section{Open questions}

Perhaps the most surprising feature of the above examples is the appearance of extra factors of one half in the normalization of some D-brane charges. Even in the case of $\mathbb{R} P^{7} \times S^{3}$ this one half meant that a D-brane wrapping $\mathbb{R} P^{3}$, which has a spin normal bundle and no $B$-flux, contains half a unit of $\mathbb{R} P^{1}$-wrapping D-brane charge. This $\mathbb{R} P^{1}$ brane corresponds to a bundle with all vanishing Chern classes, but the corresponding term in the spectral sequence was nontrivial. Perhaps one may reconstruct the nontrivial spectral sequence element by combining the Chern class and the $\sqrt{\hat{A}}$ terms of the Dbrane effective action in such a way as to preserve this torsion. After all the worldvolume action of the brane does presumably contain the information about lower dimensional brane charges. The other possibility is that the factor of one half is always present, in which case one needs to learn how to make this division by two canonical.

Another puzzling fact is the inclusion of the half D2-brane charge in the $\mathbb{Z}$-valued $K$-theory. The spacetime is compact and so the integral of $d G_{6}$ must be zero and so the D2 charge is precisely determined by the FreedWitten type terms. The D2 charge is $T$-dual to the $\mathbb{Z}_{2}$ valued D3 charge and so is odd, but different odd values are related by $d G_{6}$ charged D2 branes, and so only one odd value is consistent. One may then ask what determines this value, that is, what does the nontrivial element of $\mathbb{Z}_{2}$ lift to once the $\mathbb{Z}_{2}$ is included in $\mathbb{Z}$ by the extension problem. This is the same as choosing the integral part of $B$. One may ask whether this choice is merely a choice of gauge, or an observable. But if it is observable then conceivably it corresponds to a choice on the original product of projective spaces, where there is not obviously any $\mathbb{Z}$-valued ambiguity. Even multiples of D2's are dual to D1's in the original picture, and so it is reasonable to conjecture that the number of pairs of D2's is determined by whatever consistency condition 
determines the D1 charge. Again it would be useful to find a formula for D1 charge like the formula that we propose for D3 charge.

In [38] it was shown that the DMW anomaly, given by $W_{7}$, is absent if the partition function is defined in (complex oriented) elliptic cohomology. The study was done for the partition function of the fields in the absence of branes, in the same spirit as DMW. In the current paper we chose to include D-branes in the analysis and that has led us to propose a modification of the $W_{7}$ condition due to the presence of D2-branes. It would be interesting to see how the corresponding discussion in [38] would be extended.

One main question is to what extent $S$-duality is compatible with (twisted) $K$-theory. In [39] this was studied starting from the conjecture in [4], proven in [19], that in the absence of D3-brane charge

$$
H \cup H+G_{3} \cup H+G_{3} \cup G_{3}+P=0 .
$$

In this context, it was shown in [39] that the $H \cup H$ term causes an affine twisting, but subsequently that it is inconsistent in the framework of any $K(\mathbb{Z}, 2)$-twisting. Further, the $P$ term was also shown to cause affine twisting implying that in order to have $S$-duality in type IIB in ten dimensions, twistings by $H$ must be accompanied by some higher-dimensional nontrivial twisting. Such higher twistings, as pointed out in [39], as well as the $P$ term, in fact correspond to constructing a Postnikov tower of a classifying space. In [39], it was proposed that this space should correspond to a generalized cohomology theory, which was conjectured to be a form of elliptic cohomology. It is, however, alternately possible to construct this Postnikov tower directly, identifying all the homotopy groups (such as $\mathrm{P}$ and higher twistings), and the Postnikov invariants between them, which correspond to equations such as (7.1). More on this will appear in the future. However, it is also evident from that work that in looking at the problem from a higher-dimensional perspective, e.g. in twelve dimensions, one seems to inevitably need elliptic cohomology. (Some aspects of elliptic cohomology in 12 dimensions has been discussed recently in [40].)

There are a number of directions for future research. For example this approach may be used to try to construct the Atiyah-Hirzebruch differentials in general. While $S$-dual anomalies require that we restrict attention to 10 dimensions, we found a term in $d_{5}$ in the untwisted case where the $S$-dual effects were not important. In fact it seems as though there is a consistent truncation of the formalism in which the $S$-dual gauge transformations and $S$-dual Freed-Witten anomalies are ignored, so that one may consider more dimensions and thus use the worldvolume D-brane charges to construct the higher differentials. This truncation appears automatically in the context of conformal field theories and so this approach corresponds to the fact that 
we may consider conformal field theories with targets of dimension greater than ten describing open strings whose possible boundary conditions will be classified by the desired $K$-classes.

If we go beyond this approximation then string theory provides modifications of $K$-theory which still have not been identified. For example the inclusion of $S$-duality seems to lead to an infinite family of $K$-theories that are related by a set of $S L(2, \mathbb{Z})$ transformations. The full supergravity also has the relations

$$
G_{p}=* G_{10-p}
$$

where the Hodge dual $*$ is generally irrational and so generically at most half of the RR field strengths are integral at a time. An analogy with other systems, such as the chiral scalar in 2 dimensions, suggests that this means we need to quantize $K$-theory such that only half of the Chern characters are defined at a time. Proposals for these two variations of $K$-theory will appear elsewhere.

\section{Acknowledgment}

We are very grateful to G. Moore and E. Witten for extensive help with this project and also to C. Douglas and H. Miller for explaining the role of secondary cohomology operations. P.B., V.M. and H.S. are supported, in part, by the Australian Research Council, and J.E. is partially supported by IISN-Belgium (convention 4.4505.86), by the "Interuniversity Attraction Poles Program - Belgian Science Policy" and by the European Commission FP6 programme MRTN-CT-2004-005104, in which he is associated to V.U. Brussel. B.J. is partially supported by the European Commission RTN program MRTN-CT-2004-005104. J.E. and B.J. would like to thank the University of Adelaide for hospitality while this work was in progress. B.J. would also like to thank the INFN Torino for hospitality and J.E. would like to thank Harvard University. H.S. would like to thank the Shanghai Institute for Advanced Studies and the University of Science and Technology of China for hospitality during the intermediate stages of this project.

\section{Appendix}

The real projective space $\mathbb{R} P^{n}$ is the quotient space of the sphere $S^{n}$ under the action of $\mathbb{Z}_{2}$ generated by antipodal maps $x \mapsto-x$. In particular, $\mathbb{R} P^{1}$ is just the circle $S^{1}$. Since each hemisphere in $S^{n}$ is disjoint from its antipodal image, the $\mathbb{Z}_{2}$ action is a covering space action. Since $S^{n}$ is simply connected 
for $n \geq 2$, the covering $S^{n} \rightarrow \mathbb{R} P^{n}$ gives the fundamental group $\pi_{1}\left(\mathbb{R} P^{n}\right)=$ $\mathbb{Z}_{2}$ for $n \geq 2$. The generator of this group is any loop obtained by projecting a path in $S^{n}$ connecting two antipodal points. As a consequence, one has the first homology group $H_{1}\left(\mathbb{R} P^{n}\right)=\mathbb{Z}_{2}$.

Let $L=S^{n} \times \mathbb{R} / \mathbb{Z}_{2}$ be the real classifying line bundle over $\mathbb{R} P^{n}$ and let $x=w_{1}(L)$ generate $H^{1}\left(\mathbb{R} P^{n} ; \mathbb{Z}_{2}\right)=\mathbb{Z}_{2}$. The tangent bundle of $\mathbb{R} P^{n}$ is

$$
T\left(\mathbb{R} P^{n}\right) \oplus 1=(n+1) L .
$$

The Stiefel-Whitney classes are given as follows. Let

$$
\left(\begin{array}{c}
n \\
i
\end{array}\right)_{2}=\frac{n !}{i !(n-i) !} \quad \bmod 2
$$

be the binomial coefficient reduced modulo 2 (since we are dealing with the Steenrod algebra). Then the formula for the Stiefel-Whitney classes for $\mathbb{R} P^{n}$ is

$$
w_{i}\left(\mathbb{R} P^{n}\right)=\left(\begin{array}{c}
n+1 \\
i
\end{array}\right)_{2} x^{i}
$$

Of interest are the first and the second Stiefel-Whitney classes, which characterize whether the manifold is orientable and spin, respectively. So for $i=1,2$, we have

$$
\begin{aligned}
& w_{1}\left(\mathbb{R} P^{n}\right)=(n+1) x, \\
& w_{2}\left(\mathbb{R} P^{n}\right)=\frac{1}{2} n(n+1) x^{2},
\end{aligned}
$$

keeping in mind that the coefficients are taken modulo two. In order for the manifold to be orientable, $w_{1}$ has to vanish, which implies that $n$ must be odd, as it is always in this note. Next, the spin condition is that both $w_{1}$ and $w_{2}$ vanish. This implies that $n=4 k+3$. In particular, $\mathbb{R} P^{3}$ and $\mathbb{R} P^{7}$, which we use, are spin manifolds. In this case one can calculate the first cohomology with $\mathbb{Z}_{2}$ coefficients, $H^{1}\left(\mathbb{R} P^{4 k+3}, \mathbb{Z}_{2}\right)$, to be $\mathbb{Z}_{2}$, which implies that there are two inequivalent spin structures on $\mathbb{R} P^{3}$ and $\mathbb{R} P^{7}$. Of course we also know that $\mathbb{R} P^{1}=S^{1}$ which is spin and also has two spin structures (Ramond and Neveu-Schwarz). What about $\mathbb{R} P^{4 k+1}$ ? Again, inspecting the formulae, one notices that in this case $w_{1}$ vanishes but $w_{2}$ is the reduction of an integral class. This is the $\operatorname{spin}^{c}$ condition. In particular, in this paper we used the fact that $\mathbb{R} P^{5}$ is $\operatorname{spin}^{c}$.

In the text we are interested in products of projective spaces. Note that the product of two orientable manifolds is also orientable, the product of two spin manifolds is also spin, and the product of two $\operatorname{spin}^{c}$ manifolds is also $\operatorname{spin}^{c}$. 
One can also see that, besides the question of spin, there are other differences between $\mathbb{R} P^{5}$ and $\mathbb{R} P^{n}$ for $n=1,3,7$. One can see such a difference in the context of (complex) $K$-theory. As above, if $L_{\mathbb{C}}$ is the corresponding complex line bundle, then one has $\left(T\left(\mathbb{R} P^{n}\right) \oplus 1\right) \otimes \mathbb{C}=2 k \cdot L_{\mathbb{C}}$, where $n=2 k-1$. The reduced $K$-theory $\widetilde{K}\left(\mathbb{R} P^{n}\right)$ is a cyclic group of order $2^{k-1}$ which is generated by $x=\left[L_{\mathbb{C}}\right]-1$. Thus the bundle $T\left(\mathbb{R} P^{n}\right) \oplus 1$ is trivial if $2^{k-1}$ divides $2 k$. This implies that $k=1,2,4$ or equivalently that $n=1,3,7$. Therefore we have the important result that $\mathbb{R} P^{1}, \mathbb{R} P^{3}$ and $\mathbb{R} P^{7}$ are parallelizable, the same way that their double covers $S^{1}, S^{3}$ and $S^{7}$ are. This result can also be deduced from the quaternion and octonion multiplication. An important consequence of this is that all their characteristic classes are zero.

\section{References}

[1] R. Minasian and G. Moore, K-theory and Ramond-Ramond charge, J. High Energy Phys. 11 (1997), 002; arXiv:hep-th/9710230.

[2] E. Witten, D-branes and K-theory, J. High Energy Phys. 12 (1998), 019; arXiv:hep-th/9810188.

[3] P. Bouwknegt and V. Mathai, D-branes, B-fields and twisted K-theory, J. High Energy Phys. 03 (2000), 007; arXiv:hep-th/0002023.

[4] D.-E. Diaconescu, G. Moore and E. Witten, E8 gauge theory, and a derivation of K-theory from M-theory, Adv. Theor. Math. Phys. 6 (2003), 1031-1134; arXiv:hep-th/0005090.

[5] J. Evslin, Twisted K-theory from monodromies, J. High Energy Phys. 05 (2003), 030; arXiv:hep-th/0302081.

[6] D. Marolf, Chern-Simons terms and three notions of charge, in Quantization, Gauge Theory and Strings, eds. A. Semikhatov et al World Scientific, Moscow 2001; arXiv:hep-th/0006117.

[7] S.B. Giddings, S. Kachru, and J. Polchinski, Hierarchies from fluxes in string compactifications, Phys. Rev. D66 (2002), 106006; arXiv: hep-th/0105097.

[8] A. Hanany and E. Witten, Type IIB superstrings, BPS monopoles, and three-dimensional gauge dynamics, Nucl. Phys. B492 (1997), 152-190, arXiv:hep-th/9611230.

[9] D. Freed and E. Witten, Anomalies in string theory with D-branes, Asian J. Math. 3 (1999), 819; arXiv: hep-th/9907189.

[10] E. Witten, Baryons and branes in Anti de Sitter space, J. High Energy Phys. 07 (1998), 006; arXiv:hep-th/9805112. 
[11] C.M. Hull and P.K. Townsend, Unity of superstring dualities, Nucl. Phys. B438 (1995), 109; arXiv: hep-th/9410167.

[12] C.M. Hull, String-string duality in ten dimensions, Phys. Lett. B357 (1995), 545; arXiv:hep-th/9506194.

[13] J.H. Schwarz, An $S L(2, \mathbb{Z})$ multiplet of type IIB superstrings, Phys. Lett. B360 (1995), 13; arXiv:hep-th/9508143.

[14] O. Loaiza-Brito, Instantonic branes, Atiyah-Hirzebruch spectral sequence, and $S L(2, \mathbb{Z})$ duality of $N=4 \quad S Y M$, arXiv: hep-th/0311028.

[15] I.R. Klebanov and M.J. Strassler, Supergravity and a confining gauge theory: duality cascades and $\chi S B$-resolution of naked singularities, J. High Energy Phys. 08 (2000), 052; arXiv:hep-th/0007191.

[16] J. Evslin, The cascade is a MMS instanton, arXiv:hep-th/0405210.

[17] C. Vafa and E. Witten, A one-loop test of string duality, Nucl. Phys. B447 (1995), 261-270; arXiv:hep-th/9505053.

[18] S. Sethi, C. Vafa and E. Witten, Constraints on low-dimensional string compactifications, Nucl. Phys. B480 (1996), 213-224; arXiv: hep-th/9606122.

[19] E. Diaconescu, D.S. Freed, and G. Moore, The M-theory 3-form and E8 gauge theory, arXiv:hep-th/0312069.

[20] G. Moore and E. Witten, private communication.

[21] M.J. Duff, H. Lü and C.N. Pope, $A d S_{5} \times S^{5}$ untwisted, Nucl. Phys. B532 (1998), 181-209; arXiv: hep-th/9803061.

[22] A. Hanany and B. Kol, On orientifolds, discrete torsion, branes and $M$ theory, J. High Energy Phys. 06 (2000), 013; arXiv:hep-th/0003025.

[23] P. Bouwknegt, J. Evslin and V. Mathai, T-duality: topology change from H-flux, Commun. Math. Phys. 249 (2004), 383-415; arXiv: hep-th/0306062.

[24] P. Bouwknegt, J. Evslin and V. Mathai, On the topology and H-flux of T-dual manifolds, Phys. Rev. Lett. 92 (2004), 181601; arXiv:hep-th/0312052.

[25] O. Bergman, E. Gimon and S. Sugimoto, Orientifolds, RR torsion, and K-theory, J. High Energy Phys. 05 (2001), 047; arXiv: hep-th/0103090.

[26] J. Evslin and U. Varadarajan, K-theory and S-duality: starting over from square 3, J. High Energy Phys. 03 (2003), 026; arXiv: hep-th/0112084.

[27] M. Atiyah and F. Hirzebruch, Vector bundles and homogeneous spaces, Symp. Pure Math. 3 (1961), 7-38. 
[28] V. Braun, K-theory torsion, arXiv:hep-th/0005103.

[29] L. Romans, Massive $N=2 A$ supergravity in ten dimensions, Phys. Lett. B169 (1986), 374.

[30] E. Witten, Duality relations among topological effects in string theory, J. High Energy Phys. 05 (2000), 031; arXiv:hep-th/9912086.

[31] V. Mathai and H. Sati, Some relations between twisted K-theory and $E_{8}$ gauge theory, J. High Energy Phys. 03 (2004), 016; arXiv:hep-th/0312033.

[32] J.A. Harvey, P. Hořava and P. Kraus, D-sphalerons and the topology of string configuration space, J. High Energy Phys. 03 (2000), 021; arXiv: hep-th/0001143.

[33] J. Maldacena, G. Moore and N. Seiberg, D-brane instantons and K-theory charges, J. High Energy Phys. 11 (2001), 062; arXiv:hep-th/0108100.

[34] M. Atiyah and G. Segal, Twisted K-theory, Ukr. Mat. Visn. 1 (2004), no. 3, 287-330; translation in Ukr. Math. Bull. 1 (2004), no. 3, 291-334. arXiv:math.KT/0407054.

[35] I. Raeburn and J. Rosenberg, Crossed products of continuous-trace $C^{*}$ algebras by smooth actions, Trans. Amer. Math. Soc. 305 (1988), 1-45.

[36] J. Evslin, IIB soliton spectra with all fluxes activated, Nucl. Phys. B657 (2003), 139; arXiv:hep-th/0211172.

[37] A. Sen, Tachyon condensation on the brane antibrane system, J. High Energy Phys. 08 (1998), 012; arXiv:hep-th/9805170.

[38] I. Kriz and H. Sati, $M$ theory, type IIA superstrings, and elliptic cohomology, Adv. Theor. Math. Phys. 8 (2004), 345; arXiv:hep-th/0404013.

[39] I. Kriz and H. Sati, Type IIB string theory, S-duality and generalized cohomology, Nucl. Phys. B715 (2005), 639-664. arXiv:hep-th/ 0410293.

[40] I. Kriz and H. Sati, Type II string theory and modularity, J. High Energy Phys. 08 (2005), 038. arXiv: hep-th/0501060. 